\title{
Two-photon excitation dynamics in bound two-body Coulomb systems including ac Stark shift and ionization
}

\author{
M. Haas, U. D. Jentschura, and C. H. Keitel \\ Max-Planck-Institut für Kernphysik, Saupfercheckweg 1, 69117 Heidelberg, Germany
}

N. Kolachevsky, ${ }^{*}$ M. Herrmann, P. Fendel, M. Fischer, Th. Udem, R. Holzwarth, and T. W. Hänsch

Max-Planck-Institute of Quantum Optics, Hans-Kopfermann-Straße 1, 85748 Garching, Germany

M. O. Scully

Institute for Quantum Studies and Department of Physics, Texas A\&M University, College Station, Texas 77843-4242, USA

and Princeton Institute for Materials Research, Princeton University, Princeton, New Jersey 08544-1009, USA

G. S. Agarwal

Physics Research Laboratory, Nevrangpura, Ahmedabad-380 009, India

and Department of Physics, Oklahoma State University, Stillwater, Oklahoma 74078, USA

(Received 19 January 2006; published 3 May 2006)

\begin{abstract}
One of the dominant systematic effects that shift resonance lines in high-precision measurements of twophoton transitions is the dynamic (ac) Stark shift. For suitable laser frequencies, the ac Stark shift acquires an imaginary part which corresponds to the rate of resonant one-photon ionization of electrons into a continuum state. At the current level of spectroscopic accuracy, the underlying time-dependent quantum dynamics governing the atomic two-photon excitation process must be well understood, and related considerations are the subject of the present paper. In order to illustrate the basic mechanisms in the transient regime, we investigate an analytically solvable model scenario for the population dynamics in the density matrix formalism and describe in detail how to generalize the corresponding equations of motion for individual experimental use. We also calculate the dynamic Stark shift for two-photon $S-S$ and $S-D$ transitions in bound two-body Coulomb systems and the corresponding two-photon transition matrix elements. In particular, we investigate transitions for which the $1 S$ ground state or alternatively the metastable $2 S$ state acts as the lower-energy state, and for which states with $n \leqslant 20$ represent the upper states. Relativistic and radiative corrections to the excitation dynamics, and the corresponding limitations to the accuracy of the measurements, are briefly discussed. Our considerations suggest the general feasibility of a detection mechanism, offering high quantum efficiency, based on two-step three-photon resonant ionization spectroscopy, for large classes of experimentally relevant two-photon transitions in two-body Coulomb systems.
\end{abstract}

DOI: 10.1103/PhysRevA.73.052501

PACS number(s): 31.10. $+\mathrm{z}, 31.15 .-\mathrm{p}, 06.20 . \mathrm{Jr}$

\section{INTRODUCTION}

Like the electron gyromagnetic ratio, two-photon transitions in hydrogenlike systems belong to the few cases where experimental values can be compared with quantum electrodynamic (QED) calculations with very high precision. These comparisons are the basis for verification or falsification of this fundamental theory. In view of the increasing accuracy of two-photon spectroscopic experiments, it is necessary to understand in detail the excitation dynamics and the line profile of the relevant resonances, as they can be observed under realistic experimental conditions. Experiments investigating the hydrogen $S-S$ and $S-D$ transitions are also important for the determination of fundamental constants and their possible time variation [1-3].

Concurrent with the experimental progress in this field, there has been a wealth of theoretical studies addressing sys-

*Permanent address: P. N. Lebedev Physics Institute, Leninsky prospekt 53, 119991 Moscow, Russia. tematic effects, predicting the spectral line profile and calculating atomic properties connected to these problems [4-13]. Ionization cross sections of atoms in excited states of typical two-photon transitions have been obtained in [4] as early as 1930. Very detailed studies of dynamic Stark shifts and broadening effects on the spectral line shape of two-photon transitions in hydrogen have been carried out, e.g., in [5-9]. In these investigations, a large set of values for the dynamic polarizability for transitions to highly excited states is given. For hydrogen, the emphasis has been on the metastable $2 S$ state acting as the ground state. A detailed review of twophoton transition line shapes and experiments aimed at the determination of the Rydberg constant can be found in [10].

Experiments investigating the $1 S-2 S$ transition in muonium and positronium for the first time $[11,12]$, and accurate measurements of the transition frequency [13] have been reported, extending high-precision spectroscopy to bound twobody Coulomb systems beyond atomic hydrogen. A detection scheme for muonium, based on ionization, has been both described experimentally $[13,14]$ and analyzed theoretically [8]. For the future, measurements on antihydrogen and hy- 
drogenlike ions are planned, and coherent light sources in the extreme ultraviolet, which are needed for some of these experiments, have been demonstrated recently [15].

In this paper, we reconsider the derivation of the dynamic polarizabilities and transition matrix elements for $S-S$ and $S-D$ two-photon transitions in hydrogenlike systems in a unified treatment, compiling the results in SI units for convenient experimental use. We include the dependence on the nuclear charge number $Z$, where the two-photon resonance condition for the laser frequency is maintained for different $Z$, and the dependence on the nuclear mass, so that our results are applicable to general two-body Coulomb systems, such as hydrogen, positronium, muonium, antiprotonic helium, etc. Considering the excited state $n S$ of a bound twobody Coulomb system, driven by a laser field on two-photon resonance with the $1 S-n S$ transition, absorption of one additional photon is sufficient to ionize the system. The same applies to all but $n=3$ of the $2 S-n S$ transitions and to the corresponding $S-D$ transitions. The excitation of many twophoton transitions must therefore necessarily take place in the transient regime, because the driving laser would otherwise ionize all atoms in the excited state, in the limit of infinite interaction time (neglecting recombination). Thus we consider the time-dependent quantum dynamics for $S-S$ and $S-D$ transitions, including both photoionization and spontaneous decay of the system. We obtain an analytic solution of the master equation for finite times in the special case of constant intensity, and observe some interesting features in the line shape of the transition. Straightforward generalizations of the formalism to specific setups are given, which are accessible to a numerical treatment. For different nuclear charges, the relative importance of spontaneous decay versus ionization is discussed.

Even though this work aims to facilitate experimental tests of QED, it should be stressed that calculations in the framework of nonrelativistic Schrödinger theory are currently sufficient to describe the experimental line shape for low $Z$ accurately. However, relativistic and radiative corrections to the matrix elements that enter into the line shape constitute small but interesting effects. We give explicit relativistic results for selected transitions, to indicate the orders of magnitude, and we clarify some minor inconsistencies in the literature, in particular with respect to the ionization cross section and a few numerical differences.

As a concrete case, we consider the $1 S-2 S$ hydrogen measurement [16], where the natural linewidth of the metastable $2 S$ level is $1.31 \mathrm{~Hz}$ and yet the observed spectral width of the resonance line is about a few hundred hertz. The contributions due to the time-of-flight broadening, and the ionization broadening are intertwined with the quantum dynamics of the excitation process, so that they cannot simply be added in quadrature or in any other simple algebraic way.

In short, the purpose of this paper is twofold. Our first aim is to indicate the importance of ionization in the quantum dynamics and to describe the absorption spectrum in twophoton resonance spectroscopy. The second aim is to compile results for the two-photon transition matrix elements, dynamic polarizabilities and ionization cross sections that enter into the equations of motion, keeping all factors of $\hbar, c$, and $\epsilon_{0}$ in the derivation. In combination, these ingredients provide a toolkit to readily model a large variety of experimental situations in the proper SI units.

The paper is organized as follows: In Sec. II, the quantum dynamics in terms of the optical Bloch equations is discussed. Section III is dedicated to the two-photon transition matrix elements of a variety of two-photon transitions in two-body Coulomb systems. In Sec. IV, we present the dynamic Stark shift and ionization coefficients for the same set of transitions, including a brief outline of radiative and relativistic corrections in Sec. IV D. Finally, conclusions are drawn in Sec. V.

\section{QUANTUM DYNAMICS}

\section{A. Introduction}

Two-photon transitions in hydrogen and hydrogenlike ions are of special interest to high-precision spectroscopy $[16,17]$, because they allow for a suitable elimination of the first-order Doppler shift via absorption of two counterpropagating photons. In order to calculate the absorption line shape, taking into account the remaining systematic effects, it is necessary to study the time-dependent interaction process, which is the subject of this section, including the dynamic Stark effect, the ionization of the excited state, and the second-order Doppler shift. The resulting equations of motion for the atom-laser interaction can be adapted to suit different experimental setups and different two-body Coulomb systems. For the special case of constant light intensity, they are solvable analytically.

In the mentioned experiment [16], a beam of hydrogen atoms is excited by laser radiation in a linear cavity which serves to produce an amplified standing wave. The injected atoms (initially in the $1 S$ ground state) can be excited to the $2 S$ state by two-photon absorption, and the number of excited $2 S$ atoms is measured as a function of laser frequency after a certain interaction time. This signal, referred to as "the line shape" below, is generated by an ensemble of atoms, characterized by the experimental setup. Decisive parameters include (i) the spatial light intensity profile, (ii) the second-order Doppler shift, (iii) the finite light-atom interaction time, i.e., temporal intensity profile, and (iv) the varying ionization probability during the interaction. The resulting contributions of the atomic ensemble, specific to the experiment, can be taken into account in a numerical integration of the equations of motion presented in the following. For the hydrogen $1 S-2 S$ experiment, this has been carried out in a Monte Carlo approach [18].

\section{B. Basic quantum dynamics}

In this section, the basic model which we use for the excitation and ionization of two-body Coulomb systems is described. The main approximations we will make is to consider the driving laser field to be monochromatic, and to neglect the direct three-photon ionization of the ground state.

The appropriate description of excitation with a finitebandwidth laser field necessitates a treatment involving stochastic differential equations $[19,20]$ and is out of the scope of this paper. However, we would like to stress that in most 
systems, the monochromatic approximation is a good approximation, because the ionization of the excited state limits the total interaction time of the bound system with the laser field. As long as the finite bandwidth of the laser is small compared to the transient width of the excited state, which for reasons of principle can never be smaller than the inverse interaction time, a monochromatic laser is a good approximation. Note that for spectroscopic experiments probing a steady state, with an infinite interaction time, we would have to compare the laser linewidth to the natural linewidth instead.

Although the current paper is exclusively concerned with a laser whose linewidth is so small that it can be regarded as monochromatic for the purposes of the current investigation (see the discussion above), we would like to comment briefly on the issues regarding a finite laser linewidth. For shorttime evolution of the density matrix in the regime where the excited state population $\rho_{e e} \propto \Omega^{2} t^{2}$ ( $\Omega$ is the two-photon Rabi frequency on two-photon resonance), a simple averaging of $\rho_{e e}$ over the power spectrum of the laser can be applied [see Eq. (2.74) of [21]], but this averaging is not applicable for longer excitation times. In the latter case, one has to take into account the fact that the phase fluctuations of a laser typically constitute a stochastic process which necessitates a modification of the coherence terms in the optical Bloch equations, in the sense discussed in [22], resulting in further damping terms entering the right-hand side of Eq. (10b) below.

As an example for the monochromatic excitation, one may consider the hydrogen $1 S$ - $2 S$ experiment, where the excitation typically takes place on a submillisecond time scale. The spectral line width of the laser is on the order of $200 \mathrm{~Hz}$ at $121 \mathrm{~nm}$, which is small compared to the typical inverse interaction time of some kilohertz, while the natural linewidth of the $2 S$ state is only $1.31 \mathrm{~Hz}$.

The standard approach $[23,24]$ is to solve the density matrix equations for a two-level system. We can restrict the Hilbert space of the atom to only two relevant states, an excited state $|e\rangle$ and a ground state $|g\rangle$, because near resonance, only that two-photon transition will be driven significantly by the laser field. We will assume that the atoms are initially in $|g\rangle$ and are irradiated with the intensity $I(t)$, as observed by the atom, starting from time $t=0$. For specific considerations concerning the intensity in a standing wave, see Sec. II E below. The straightforward treatment of this interacting two-level system is extended by including decay channels due to spontaneous decay and ionization into the density matrix equations, which will turn out to be crucial ingredients.

Our starting point is the von Neumann equation [23] for the density operator $\rho$,

$$
i \hbar \frac{\partial}{\partial t} \rho=[\tilde{H}, \rho],
$$

with

$$
\begin{aligned}
\tilde{H}= & E_{g}|g\rangle\left\langle g\left|+E_{e}\right| e\right\rangle\langle e|+\frac{\hbar \Omega}{2}\left[\exp \left(i \omega_{\mathrm{L}} t\right)+\exp \left(-i \omega_{\mathrm{L}} t\right)\right]^{2} \\
& \times(|e\rangle\langle g|+| g\rangle\langle e|)
\end{aligned}
$$

$$
\begin{aligned}
& E_{g}=h \nu_{g}+h \Delta \nu_{\mathrm{ac}}(g), \\
& E_{e}=h \nu_{e}+h \Delta \nu_{\mathrm{ac}}(e) .
\end{aligned}
$$

The tilde on $\widetilde{H}$ signifies that the Hamiltonian is restricted to the two atomic states under consideration, as opposed to the following sections. The angular frequency of the laser is denoted by $\omega_{\mathrm{L}}$. Generally, throughout the paper, we will use both the symbols $\omega$ and $\Omega$ for angular frequencies (measured in $\mathrm{rad} / \mathrm{s}$ ) appearing in the argument of exponential functions of the form $\exp (i \omega t)$, and $\nu$ for frequencies as measured in hertz (compatible with the international unit system SI).

The energies of the excited state $E_{e}$ and ground state $E_{g}$ already include the dynamic Stark effect, expressed by the respective frequency shift $\Delta \nu_{\mathrm{ac}}$. As will be presented in detail in Sec. IV, these frequency shifts are proportional to the intensity $I(t)$ of the exciting laser field, and in accordance with [6], the ac Stark coefficient $\beta_{\mathrm{ac}}$ is defined as

$$
\Delta \nu_{\mathrm{ac}}(g)=\beta_{\mathrm{ac}}(g) I(t),
$$

and likewise for the excited state. The ac Stark coefficients are calculated in Sec. IV and listed in SI units in Tables IV-VII below. Further, we define the two-photon Rabi frequency $\Omega$ as

$$
\Omega=2\left(2 \pi \beta_{g e}\right) I(t),
$$

and due to the two-photon nature of the excitation process, $\Omega$ is also proportional to the light intensity, rather than to the electric field amplitude, as is the case for one-photon dipoleallowed transitions. Section III treats the calculation of the two-photon transition matrix elements $\beta_{g e}$ and lists the results for a set of transitions in Tables II and III below.

For the description of the population dynamics of the system, it is useful to factor out a fast-oscillating term of the off-diagonal elements of the density matrix in the equations of motion (1). We denote the transformed density matrix elements by a prime and define

$$
\begin{gathered}
\rho_{g g}^{\prime}:=\rho_{g g}, \quad \rho_{g e}^{\prime}:=\rho_{g e} \exp \left(-i 2 \omega_{\mathrm{L}} t\right), \\
\rho_{e e}^{\prime}:=\rho_{e e}, \quad \rho_{e g}^{\prime}:=\rho_{e g} \exp \left(i 2 \omega_{\mathrm{L}} t\right) .
\end{gathered}
$$

This corresponds to a transformation into the interaction picture, but with a phase factor of $\exp \left(-i 2 \omega_{\mathrm{L}} t\right)$ instead of $\exp \left(-i \omega_{e g} t\right)$ for the coherence $\rho_{g e}$. Note that the diagonal elements of the density matrix, representing the population, are invariant under this transformation. The resulting equations of motion (EOMs) for the matrix elements of the transformed density operator $\rho^{\prime}$ then contain both slowly varying terms, which determine the time scale of the population dynamics, and terms oscillating with $\pm 2 \omega_{\mathrm{L}}$ and $\pm 4 \omega_{\mathrm{L}}$. We can now employ the rotating-wave approximation, dropping the terms oscillating at these optical frequencies, thereby neglecting the Bloch-Siegert shifts [25]. Relative to the ac Stark shift, the Bloch-Siegert shift in a two-photon transition is suppressed by a factor of $\Omega / \omega_{\mathrm{L}}$, which is on the order of $10^{-10}$ for realistic intensities considered here. This is a small effect, compared with the relativistic, radiative, and field configuration corrections discussed below. 
The spontaneous decay and the ionization of the excited state can now be taken into account by adding

$$
\begin{aligned}
\rho_{\text {relax }}^{\prime}= & -\left(\gamma_{\mathrm{i}}+\gamma_{\mathrm{s}}\right) \rho_{e e}^{\prime}|e\rangle\left\langle e\left|+\gamma_{\mathrm{s}} \rho_{e e}^{\prime}\right| g\right\rangle\langle g| \\
& -\frac{\gamma_{\mathrm{i}}+\gamma_{\mathrm{s}}}{2}\left(\rho_{g e}^{\prime}|g\rangle\left\langle e\left|+\rho_{e g}^{\prime}\right| e\right\rangle\langle g|\right)
\end{aligned}
$$

to the right-hand side of Eq. (1), after performing the transformation (7). The spontaneous decay rate is denoted by $\gamma_{\mathrm{s}}$, while $\gamma_{\mathrm{i}}$ represents the rate with which the excited state is depopulated due to one-photon resonant ionization:

$$
\gamma_{\mathrm{i}}=2 \pi \beta_{\mathrm{ioni}}(e) I(t)
$$

All transition rates in this paper, denoted by $\gamma$ with an appropriate index, are given in angular frequency units $\mathrm{rad} / \mathrm{s}$. In cases where the decay $\gamma$ is the governing broadening mechanism this translates into a linewidth (Lorentzian full width at half maximum) of $\Delta \nu_{\gamma}=\gamma / 2 \pi$. The ionization coefficient $\beta_{\text {ioni }}$ is closely connected to the dynamic Stark effect which is discussed in Sec. IV. Essentially, the dynamic Stark coefficient is a complex quantity, the real part yielding $\beta_{\mathrm{ac}}$, and the imaginary part determining $\beta_{\text {ioni }}$. The ionization rate (9) is included into the equations of motion in analogy with the spontaneous decay rate, with one important difference. In the particular case of the $1 S-2 S$ experiment, the atomic density is very low, in order to avoid collisional effects on the transition frequency. Therefore, the recombination probability for protons and electrons to form again a hydrogen atom in the ground state is extremely small. Consequently, we do not include a recombination term into $\rho_{\text {relax }}^{\prime}$. In cases where recombination by radiative or three-body processes cannot be neglected, the respective rates are described by the principle of detailed balance (see, e.g., pp. 102 and 151 of [26]).

In the cases where the lower level $|g\rangle$ is the $2 S$ state, the excited state $n S$ or $n D$ can decay spontaneously into several levels, which in turn cascade (i) to the metastable $2 S$ state, dominantly with an effective rate $\gamma_{\mathrm{s}}^{2 S}$ and (ii) to the $1 S$ ground state, with an effective rate $\gamma_{\mathrm{s}}^{1 S}$. If the decay cascade ends in $|1 S\rangle$, the population is lost for the dynamics of the considered two-level system, and can therefore be treated as an additional intensity-independent ionization rate: $\gamma_{\mathrm{i}} \rightarrow 2 \pi \beta_{\text {ioni }}(e) I+\gamma_{\mathrm{s}}^{1 \mathrm{~S}}$.

We arrive at the following set of equations, which are equivalent to, e.g., Eq. (8) of Ref. [8], in the case where $\gamma_{\mathrm{s}}=0$ :

$$
\begin{gathered}
\frac{\partial}{\partial t} \rho_{g g}^{\prime}=-\Omega \operatorname{Im}\left(\rho_{g e}^{\prime}\right)+\gamma_{\mathrm{s}} \rho_{e e}^{\prime}, \\
\frac{\partial}{\partial t} \rho_{g e}^{\prime}=-i \Delta \omega \rho_{g e}^{\prime}+i \frac{\Omega}{2}\left(\rho_{g g}^{\prime}-\rho_{e e}^{\prime}\right)-\frac{\gamma_{\mathrm{i}}+\gamma_{\mathrm{s}}}{2} \rho_{g e}^{\prime}, \\
\frac{\partial}{\partial t} \rho_{e e}^{\prime}=\Omega \operatorname{Im}\left(\rho_{g e}^{\prime}\right)-\left(\gamma_{\mathrm{i}}+\gamma_{\mathrm{s}}\right) \rho_{e e}^{\prime},
\end{gathered}
$$

with the definition for the excitation detuning

$$
\Delta \omega=2 \pi \Delta \nu=2 \omega_{\mathrm{L}}-2 \pi \nu_{e g}-2 \pi\left[\Delta \nu_{\mathrm{ac}}(e)-\Delta \nu_{\mathrm{ac}}(g)\right]
$$

Here, the absolute frequency of the unperturbed transition is $\nu_{e g}$. Whereas the first-order Doppler shift is often canceled by the use of two counterpropagating beams, the secondorder Doppler shift of an atom moving with velocity $v$, like any other single-particle frequency shift, can be included into the excitation detuning by adding

$$
\Delta \omega_{\mathrm{D} 2}=\left(2 \pi \nu_{e g}\right) \frac{1}{2} \frac{v^{2}}{c^{2}}
$$

to the right-hand side of Eq. (11). At this point, we would like to remark that the EOMs (10) are similar to the case of a two-level system, coupled by a laser field driving a dipoleallowed one-photon transition. In fact, the main conceptual difference lies in the calculation of the transition matrix elements entering into the Rabi frequency and the dynamic Stark coefficients. In particular, the dynamic Stark effect is fundamentally different for the two-photon case, where the harmonic electric field is an off-resonant perturbation of second order, as opposed to the resonant one-photon case, where the level shift is linear in the electric field amplitude. Note also that, in contrast to one-photon transitions, the spontaneous decay rate is modified slightly in the presence of the laser field due to virtual intermediate $P$ states, even for the $1 S-2 S$ transition, as detailed in Appendix D.

\section{Analytic solution for constant intensity}

In the above form (10), for constant intensity $I(t)=I$, the EOMs are a coupled set of first-order differential equations with constant coefficients and hence are solvable analytically for all times. The somewhat lengthy expression for the full solution is given in Appendix B.

An analytic solution to Eqs. (10) without ionization $\left(\gamma_{i}=0\right)$, taken in the limit of infinite interaction time, leads to the well-known steady state of the system with a Lorentzian line shape for the excited-state population [23]. In this case, it is assumed that the population that decays out of the excited state reappears in full at the ground state. When discussing two-photon $S-S$ and $S-D$ transitions, this hardly ever happens. Even the $2 S-3 S$ transition, for which indeed $\gamma_{\mathrm{i}}=0$, does not satisfy this condition, because the population in the $3 S$ state also spontaneously decays to the $1 S$ state, mainly via $2 P$, and does not reappear in the $2 S$ state.

In this section, we will first focus on the case of vanishing spontaneous decay, $\gamma_{\mathrm{s}}=0$. This is often a good approximation when atoms or ions are excited in beams or gas cells, as opposed to trapped particles. In particular, it is a very good approximation for the hydrogen $1 S-2 S$ transition, because for typical intensities, the ionization rate dominates over the two-photon spontaneous decay rate. Later in this section, we will come back to the general solution, for cases where the spontaneous decay rate is no longer negligible (e.g., for systems with nuclear charge number $Z>1$ ).

We recall that the EOMs describe an atom at rest or moving with a constant velocity, for which the Doppler shift can be included into the detuning $\Delta \omega$. The initial state is the 
ground state, so $\rho_{g g}(t=0)=1$ and $\rho_{e e}(0)=\rho_{g e}(0)=\rho_{e g}(0)=0$. Starting from time $t=0$, the system is interacting with a monochromatic laser field of constant intensity $I$. The transient line shape, which is defined as the population in the excited $2 S$ state as a function of detuning and time, $\rho_{e e}^{\prime}(\Delta \omega, t)$, can then be expressed as

$$
\begin{aligned}
\rho_{e e}^{\prime}(\Delta \omega, t)= & \frac{4 \Omega^{2}}{G} \exp \left(-\frac{\gamma_{\mathrm{i}}}{2} t\right)\left[\sin ^{2}\left(\Omega_{1} t\right)+\sinh ^{2}\left(\Omega_{2} t\right)\right], \\
\rho_{g g}^{\prime}(\Delta \omega, t)= & \rho_{e e}^{\prime}(\Delta \omega, t)+\frac{1}{G \Omega_{1} \Omega_{2}} \exp \left(-\frac{\gamma_{\mathrm{i}}}{2} t\right) \\
& \times\left\{\Omega _ { 2 } ( 4 \Omega _ { 1 } ^ { 2 } - \Delta \omega ^ { 2 } ) \left[4 \Omega_{1} \cos \left(2 \Omega_{1} t\right)\right.\right. \\
& \left.+\gamma_{\mathrm{i}} \sin \left(2 \Omega_{1} t\right)\right]+\Omega_{1}\left(4 \Omega_{2}^{2}+\Delta \omega^{2}\right) \\
& \left.\times\left[4 \Omega_{2} \cosh \left(2 \Omega_{2} t\right)+\gamma_{\mathrm{i}} \sinh \left(2 \Omega_{2} t\right)\right]\right\},
\end{aligned}
$$

where

$$
\begin{gathered}
G=\sqrt{16 \Delta \omega^{2} \gamma_{\mathrm{i}}^{2}+\left(4 \Delta \omega^{2}+4 \Omega^{2}-\gamma_{\mathrm{i}}^{2}\right)^{2}}, \\
\Omega_{1}=\frac{1}{4 \sqrt{2}} \sqrt{G+4 \Delta \omega^{2}+4 \Omega^{2}-\gamma_{\mathrm{i}}^{2}}, \\
\Omega_{2}=\frac{1}{4 \sqrt{2}} \sqrt{G-4 \Delta \omega^{2}-4 \Omega^{2}+\gamma_{\mathrm{i}}^{2}} .
\end{gathered}
$$

The angular frequencies $\Omega_{1}$ and $\Omega_{2}$ are always real. Reassuringly, we have found that this solution has been obtained in a rather different form in $[20,27]$, but agrees with our result. These works focus on resonant multiphoton ionization and it is not surprising that the same master equations are relevant for these studies.

For the case of vanishing ionization $\beta_{\text {ioni }}=0$, we have $\Omega_{2}=0$ and we obtain the familiar Rabi oscillations with generalized Rabi frequency $\sqrt{\Delta \omega^{2}+\Omega^{2}}$ :

$$
\rho_{e e}^{\prime}(\Delta \omega, t)=\frac{1}{2} \frac{\Omega^{2}}{\Delta \omega^{2}+\Omega^{2}}\left[1-\cos \left(\sqrt{\Delta \omega^{2}+\Omega^{2}} t\right)\right],
$$

as we should. Note that the exponential decay of the excitedstate population seems to take place with only half the expected rate in Eq. (13). However, as the result describes excitation starting from the ground state and subsequent decay, the population decay rate is not simply $\gamma_{\mathrm{i}}$ for this particular solution. In comparison, the solution obtained with the same method, but with the excited state as the initial state and vanishing laser excitation $(\Omega=0)$ does in fact decay with the rate $\gamma_{i}$, independent of the detuning.

In Figs. 1-4, the analytic solution for the transient line shape of the $2 S$ population in the vicinity of the hydrogen $1 S-2 S$ two-photon resonance is illustrated, where an intensity of $2.3 \mathrm{MW} / \mathrm{m}^{2}$ is used, which is a typical magnitude in the experiment of [16]. Specifically, in Figs. 1 and 2, we investigate the influence of the ionization channel on the transient line shape for a typical interaction time of the $1 S-2 S$ experiment [16]. Observe that the inclusion of the ionization channel mainly changes the excitation efficiency while having only little effect on the spectral linewidth or on coherence

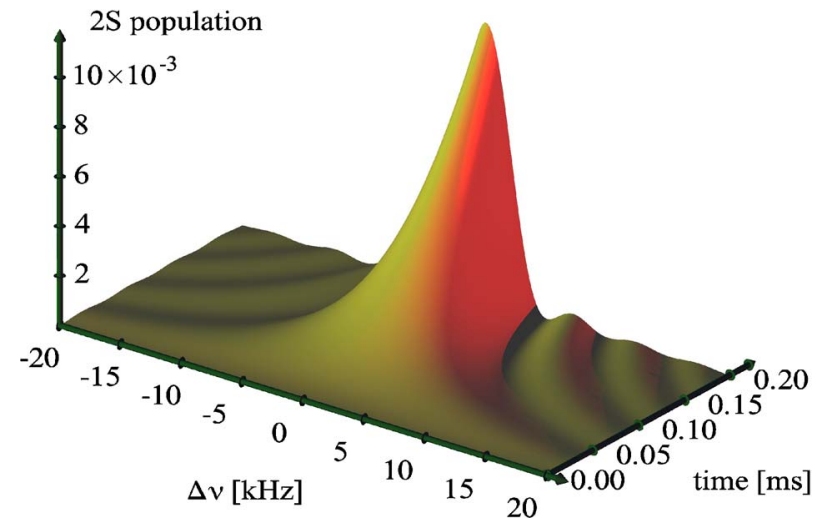

FIG. 1. (Color online) Atomic population in the $2 S$ state of hydrogen $(Z=1)$ as a function of interaction time $t$ with the laser, and of detuning $\Delta \nu$ from the $1 S-2 S$ transition frequency, as defined in Eq. (11) with $\nu_{e g} \approx 2466 \mathrm{THz}$. The laser intensity is $I=2.3 \mathrm{MW} / \mathrm{m}^{2}$, corresponding to $\Omega=2 \pi \times 169 \mathrm{~Hz}$. The initial state at $t=0$ is the $1 S$ state. In the time evolution of the system, ionization from the $2 S$ state into the continuum and spontaneous decay of the $2 S$ state have been neglected, i.e., $\rho_{e e}^{\prime}$ from Eq. (15) is plotted.

features. This is plausible, because on this time scale, which is much shorter than the Rabi oscillation time, the $2 S$ state is only populated very little.

In Figs. 3 and 4, one can observe the strong influence of the ionization on the line shape for interaction times on the order of one Rabi oscillation and longer. With ionization taken into account (Fig. 4), the excitation of the $2 S$ level is much less efficient, the coherence features (fringes) are washed out and spectral hole burning occurs, because close to zero detuning, excitation, and subsequent ionization is enhanced.

As the EOMs suggest, we obtain a symmetric line shape around $\Delta \omega=0$. However, it should be pointed out that this is no longer true for a collective signal from a thermal atomic beam. In that case, different second-order Doppler shifts according to Eq. (12), which all have the same sign, asym-

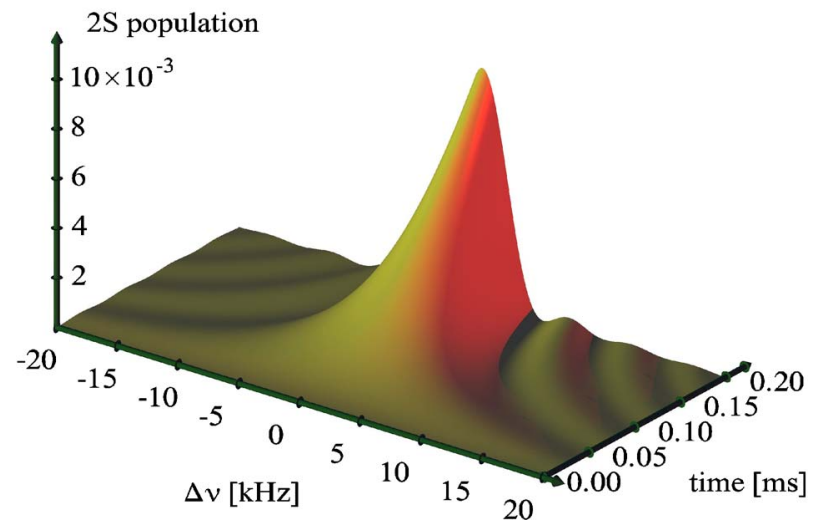

FIG. 2. (Color online) Same situation as in Fig. 1, except that in this plot, the effect of ionization was included, as in Eqs. (13) and (14). The ionization rate $\gamma_{\mathrm{i}}=2 \pi \times 276 \mathrm{~Hz}$. For the short times considered here, there is only a small difference to Fig. 1 in the total excitation efficiency, which is due to the ionization loss from the excited state. 


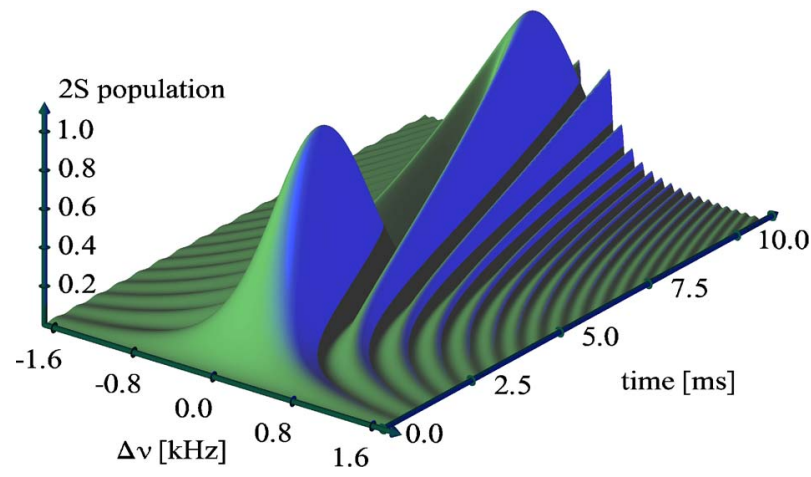

FIG. 3. (Color online) Same as Fig. 1, but for interaction times that are comparable with the Rabi oscillation time. Ionization from the $2 S$ state is not taken into account. At zero detuning, the $\sin ^{2}$-shaped Rabi oscillations with full amplitude can be observed. All the other sections with constant detuning can also be understood as the well-known Rabi oscillations with diminished amplitude and generalized Rabi frequency $\Omega_{\mathrm{R}}=\sqrt{\Delta \omega^{2}+\Omega^{2}}$ in complete analogy with one-photon transitions except that the two-photon Rabi frequency $\Omega$ as defined in Eq. (6) is proportional to the intensity, instead of the electric field amplitude.

metrically distort the line shape, depending on the atomic beam parameters. However, the discussion here also applies to a monoenergetic beam of atoms. In this case the line symmetry point is simply shifted by the second-order Doppler effect and the dynamic Stark effect. The discussion of the linewidth is more involved, because for each point in time, the line shape is different and therefore the width is not defined unambiguously in the transient regime. Nevertheless, we want to discuss qualitatively the influence of ionization, excitation intensity, and interaction time on the $\Delta \omega$ dependence of the line shape.

Consider the two $\Delta \omega$-dependent factors in the expression for the line shape (13a). The factor $1 / G$ is time independent

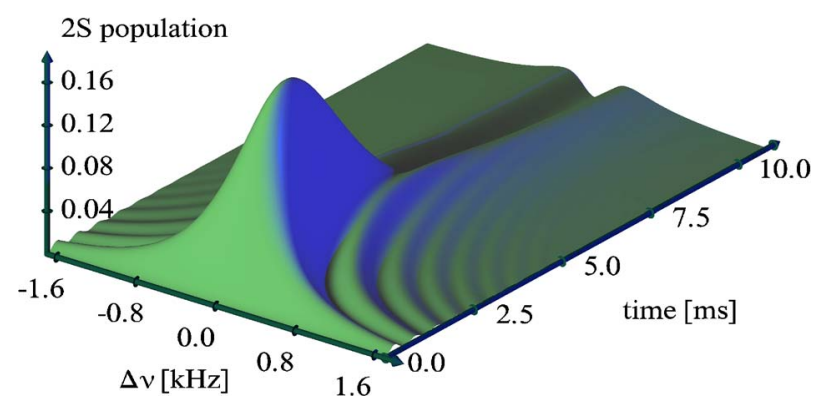

FIG. 4. (Color online) Same situation as in Fig. 3, except that here the ionization from the $2 S$ state has been properly included. The presence of the ionization channel now makes a big difference as compared to Fig. 3, because at times comparable to the Rabi oscillation time, the $2 S$ state is significantly populated and consequently the ionization probability is not negligible. The decay of the $2 S$ population, spectral hole burning, and loss of coherence can be observed in this image. The maximum population of the $2 S$ state is 0.175 [see Eq. (16)], whereas without ionization the $2 S$ population repeatedly reaches $100 \%$ for $\Delta \nu=0$. Including the $2 S$ spontaneous two-photon decay of $\gamma_{\mathrm{s}}=2 \pi \times 1.31 \mathrm{~Hz}$ does not change the plot discernibly. and indicates two contributions to the width. For increasing detuning, $1 / G$ decreases more slowly if the intensitydependent Rabi frequency $\Omega$ is large. This results in a timeindependent power-broadening contribution. Likewise, the presence of an ionization channel, quantified by the ionization rate $\gamma_{\mathrm{i}}$ adds to this width, constituting an ionization broadening.

For the time-dependent factor $\sin ^{2}\left(\Omega_{1} t\right)+\sinh ^{2}\left(\Omega_{2} t\right)$, first consider the case of vanishing ionization, $\Omega_{2}=0$ (see also Fig. 3). The remaining term $\sin ^{2}\left(\Omega_{1} t\right)$ produces fringes within the line shape that become arbitrarily narrow with increasing interaction time $t$ in the absence of any damping. However, the envelope of these fringes as well as any unfringed line shape always increase in width [28] if ionization is introduced by virtue of the $\sinh ^{2}$ term. The same holds true for power broadening described by the line shape (13). In Figs. 1 and 2 , the $\sin ^{2}(\Delta \omega) / \Delta \omega^{2}$ type of line shape, which is characteristic for the sudden turn-on of the excitation, can be recognized.

Comparing the detuning ranges of Figs. 1 and 2 with Figs. 3 and 4, one also observes that the width of the central peak decreases for increasing interaction time. This is observable in the experiment as a time-of-flight-dependent broadening. On two-photon resonance $(\Delta \nu=0)$ and for short interaction times, the $2 S$ population initially grows proportionally to $t^{2}$. The maximum excited-state population $\rho_{e e}^{\prime}(\max )$, which occurs at zero detuning $\Delta \omega=0$, reads

$$
\rho_{e e}^{\prime(\max )}=\exp \left(-\frac{\beta_{\text {ioni }} \arccos \left(\frac{\beta_{\text {ioni }}^{2}}{8 \beta_{g e}^{2}}-1\right)}{\sqrt{16 \beta_{g e}^{2}-\beta_{\text {ioni }}^{2}}}\right),
$$

and is independent of the intensity of the laser field. Note that for the strongly damped case where $\beta_{\text {ioni }}>4 \beta_{g e}$, both the arccos and the square root are complex valued, but the result remains real. If spontaneous decay is included into the dynamics, as described below, the peak excited state population $\rho_{e e}^{\prime}{ }^{(\text {peak })}$ is always less than given in Eq. (16). Expression (16) then gives the high-intensity limit for $\rho_{e e}^{\prime}$ (peak), where ionization dominates over the spontaneous decay.

One can hardly overemphasize that the steady state of the density matrix equation (10) is the one where the entire atomic population is in the ionized state. For a two-level system without ionization, in the steady state the population depends on the driving laser frequency in the form of a Lorentz curve [23]. Obviously, the ionization term changes this property drastically.

\section{Quantum dynamics with spontaneous decay}

In the following, we will focus on systems, in which the spontaneous decay channel is no longer negligible. As concrete examples, we will treat the $1 S-2 S$ and the $1 S-3 S$ transitions in systems where the nuclear charge number is not restricted to $Z=1$. Consider the $Z$ scaling of the spontaneous decay rates, listed in Table I (see, e.g., [29-31] and pp. 266267 in [32]). One-photon spontaneous decay rates of dipoleallowed transitions are denoted by $\gamma_{\mathrm{s}}^{1 \gamma}$; for two-photon spontaneous decay rates, we write $\gamma_{\mathrm{s}}^{2 \gamma}$. The ionization rate 
TABLE I. Spontaneous decay rates (angular frequency) relevant to the description of the quantum dynamics of the $1 S-2 S$ and $1 S-3 S$ transition in hydrogenlike systems with nuclear charge number $Z$.

\begin{tabular}{lc}
\hline \hline$\gamma_{\mathrm{s}}^{1 \gamma}(3 S \rightarrow 2 P)$ & $6.32 \times 10^{6} \mathrm{~s}^{-1} Z^{4}$ \\
$\gamma_{\mathrm{s}}^{1 \gamma}(2 P \rightarrow 1 S)$ & $6.25 \times 10^{8} \mathrm{~s}^{-1} Z^{4}$ \\
$\gamma_{\mathrm{s}}^{2 \gamma}(3 S \rightarrow 1 S)$ & $2.08 \mathrm{~s}^{-1} Z^{6}$ \\
$\gamma_{\mathrm{s}}^{2 \gamma}(3 S \rightarrow 2 S)$ & $6.45 \times 10^{-2} \mathrm{~s}^{-1} Z^{6}$ \\
$\gamma_{\mathrm{s}}^{2 \gamma}(2 S \rightarrow 1 S)$ & $8.23 \mathrm{~s}^{-1} Z^{6}$ \\
\hline
\end{tabular}

coefficient $\beta_{\text {ioni }}$ scales with $Z^{-4}$ [see Eq. (43) below]; therefore the ratio $R$ of the spontaneous decay rate and the ionization rate $\gamma_{i}$, as defined in (9), scales as

$$
R^{1 \gamma}=\frac{\gamma_{\mathrm{s}}^{1 \gamma}}{\gamma_{\mathrm{i}}} \propto Z^{8}, \quad R^{2 \gamma}=\frac{\gamma_{\mathrm{s}}^{2 \gamma}}{\gamma_{\mathrm{i}}} \propto Z^{10},
$$

for a given laser intensity. Recall that in hydrogen, for typical intensities, the spontaneous decay rate is small compared to the ionization rate, i.e., $R^{2 \gamma} \ll 1$. In contrast, for the $1 S$ - $2 S$ transition in hydrogenlike helium $(Z=2)$ and the same intensity of $2.3 \mathrm{MW} / \mathrm{m}^{2}$, both rates are of comparable magnitude, with $R^{2 \gamma} \approx 1.6$. The simplified form of the analytic solution (13) is therefore no longer valid, and even qualitative discussions of the analytic form of the full solution for the line shape (see Appendix B) are complicated. Nevertheless, one can plot the full solution (B1), and Figs. 5 and 6 show the transient line shape of the excited $\mathrm{He}^{+}$ion, irradiated with a $\mathrm{cw}$ laser of intensity $2.3 \mathrm{MW} / \mathrm{m}^{2}$. This intensity is chosen to simplify the comparison with the hydrogen plots, although a $\mathrm{cw}$-laser source at $61 \mathrm{~nm}$ does not yet exist.

In Fig. 5 one can observe that on a time scale comparable to the inverse spontaneous decay rate, the line shape evolves into a "quasi-steady-state" of approximate Lorentzian profile. Only on a longer time scale does ionization become impor-

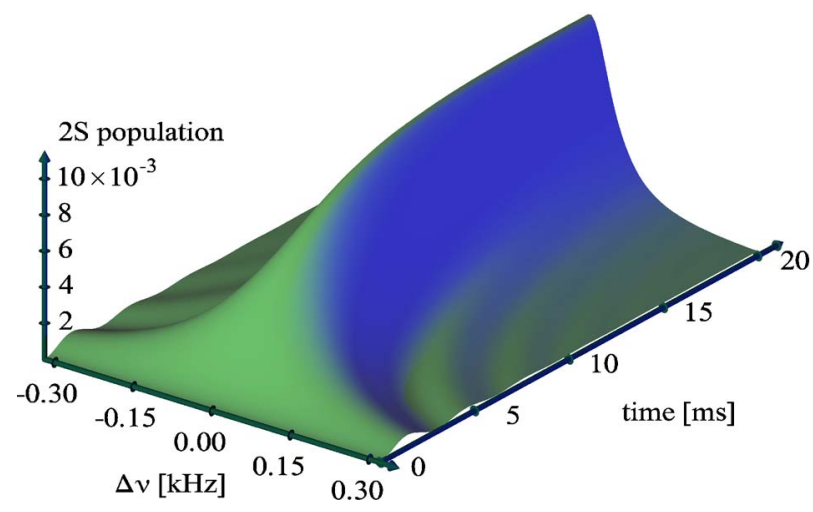

FIG. 5. (Color online) $2 S$ population in a $\mathrm{He}^{+}$ion, as a function of detuning [defined in Eq. (11)] and interaction time with the laser driving the $1 S-2 S$ transition. Ionization and spontaneous twophoton decay of the $2 S$ state are taken into account. A constant intensity of $2.3 \mathrm{MW} / \mathrm{m}^{2}$ is assumed and the ion is in the $1 S$ ground state at time $t=0$. On the time scale considered, the system evolves into a quasi-steady-state with approximate Lorentzian line shape.

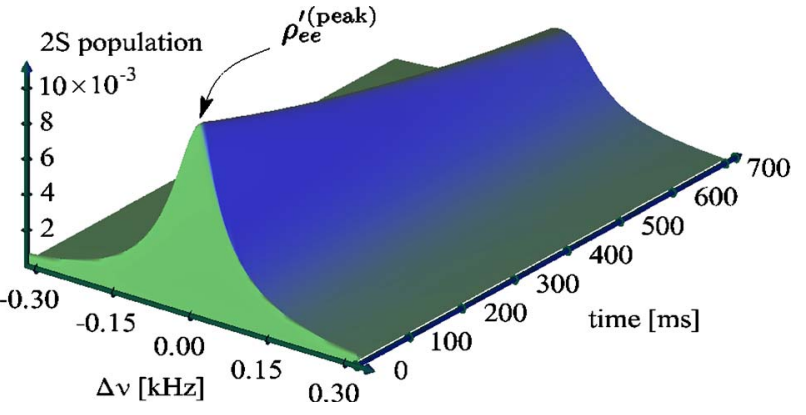

FIG. 6. (Color online) $2 S$ population dynamics including twophoton spontaneous decay in a $\mathrm{He}^{+}$ion as in Fig. 5, but on a much longer time scale. Here the decay of the population due to ionization is visible. Note that the effective population loss is by far smaller than the ionization rate $\gamma_{\mathrm{i}}=2 \pi \times 17.3 \mathrm{~Hz}$ (corresponding to a characteristic ionization time of $58 \mathrm{~ms}$ ), because the quasi-steadystate population of the excited state is small. The peak population in the excited state is $10.6 \times 10^{-3}$ for the considered intensity of $2.3 \mathrm{MW} / \mathrm{m}^{2}$. The steep rise before $t=20 \mathrm{~ms}$ is shown in more detail in Fig. 5.

tant, and the excited state is significantly depopulated (see Fig. 6).

The characteristic decay time of the excited $2 S$ population is by far longer than the inverse ionization rate $2 \pi / \gamma_{i}$. The reason for this seemingly unintuitive behavior is that the laser field continuously drives the system toward the steady state, which it would reach for $\gamma_{i}=0$. When the excited state is depopulated by ionization, population is again transferred into the excited state from the ground state, which is much more populated than the excited state for the intensity considered.

For $\gamma_{\mathrm{s}} \neq 0$, the peak population of the excited state, $\rho_{e e}^{\prime}$ (peak), occurring in the transient dynamics, is a function of the intensity of the driving laser, when spontaneous decay is included in the EOMs. For $\mathrm{He}^{+}$, this dependence is shown in Fig. 7. For large intensities, the peak population approaches the maximum value given in Eq. (16).

By calculating the population in the continuum $P$ state, $1-\rho_{g g}^{\prime}(\Delta \omega, t)-\rho_{e e}^{\prime}(\Delta \omega, t)$, one obtains the probability of ionization via the two-photon resonant excited state as a func-

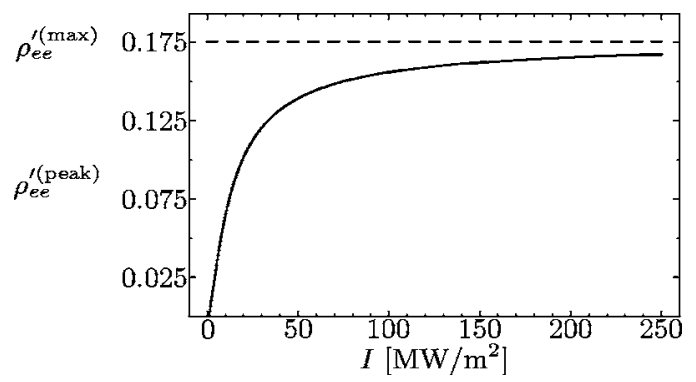

FIG. 7. Peak $2 S$ population in $\mathrm{He}^{+}$, as a function of intensity, including spontaneous decay (solid line) and without spontaneous decay (dashed line), which is equal to $\rho_{e e^{\prime}(\max )}$ in Eq. (16), evaluated for the $1 S-2 S$ transition. For increasing intensity, the ionization rate eventually becomes large compared to the spontaneous decay rate, and the peak population increases, approaching the maximum $\rho_{e e^{\prime}}^{(\max )}$. 


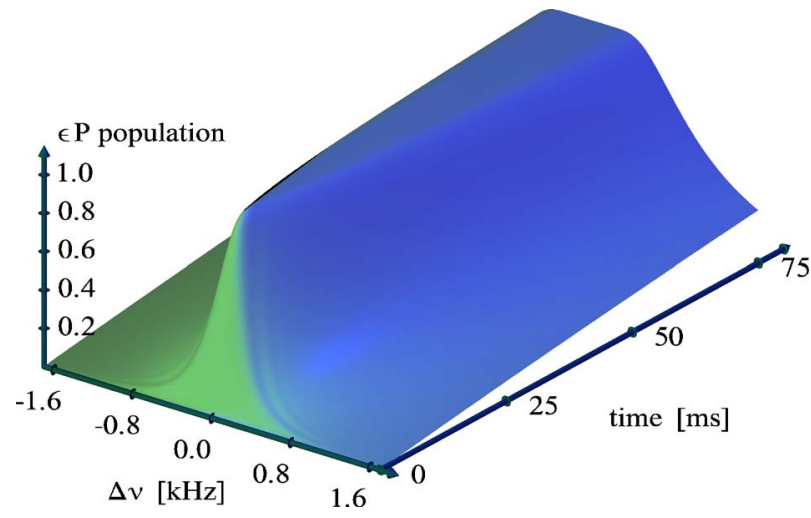

FIG. 8. (Color online) Population in the continuum $P$ state as a function of detuning [defined in Eq. (11)] and interaction time with the laser, driving the hydrogen $1 S-2 S$ transition, including spontaneous two-photon decay of the intermediate $2 S$ state with $\gamma_{\mathrm{s}}=2 \pi \times 1.31 \mathrm{~Hz}$. A constant intensity of $2.3 \mathrm{MW} / \mathrm{m}^{2}$ is assumed and the atom is in the $1 S$ ground state at $t=0$.

tion of detuning and time, if $|g\rangle$ is the $1 S$ state. In Fig. 8, we plot this $\varepsilon P$ population from the full solution (B1) of the EOMs (10) for the $1 S-2 S$ transition in hydrogen, and an intensity of $2.3 \mathrm{MW} / \mathrm{m}^{2}$, as before. Note that in this case of nonvanishing laser intensity and ionization $\gamma_{i} \neq 0$, the steady state can be defined as the completely ionized atom, while for $\gamma_{i}=0$, a regular steady state with a certain population distribution between the excited and ground states exists. The limit $\gamma_{\mathrm{i}} \rightarrow 0$ is therefore nonuniform, in the sense that the steady state for $\gamma_{i} \neq 0$ does not tend to the steady state of the case $\gamma_{i}=0$. As a result, for increasing interaction time, the detuning range in which the atomic population is completely ionized is increasing in width, as can be observed in Fig. 8. For a precision experiment relying on the detection of the free electrons, or alternatively the ionic cores, this means that the interaction time has to be chosen carefully in order to obtain a signal of minimal width. Using a detection scheme for charged particles instead of excited atoms has the advantage of a much higher detection efficiency.

For the $1 S-2 S$ transition, the direct two-photon spontaneous decay rate $\gamma_{\mathrm{s}}$ is the only spontaneous decay channel (see also Appendix D). For the $1 S-3 S$ transition the dominating decay takes place via the real intermediate $2 P$ state, because the one-photon rates are orders of magnitude larger than the two-photon rates (see Table I and Fig. 9).

Strictly speaking, it would be necessary to introduce a new set of EOMs including the real intermediate $2 P$ level. However, the $2 P$ state is not resonantly coupled to some other state by the laser field and is populated only by incoherent decay. In addition, the decay rate out of $2 P$ is 100 times larger than the decay rate into it. We can therefore approximate the quantum dynamics of a $1 S-3 S$ transition by the EOMs (10), if we use an effective decay rate from $3 S$ to $1 S$ which equals $\gamma_{\mathrm{eff}}=\gamma_{\mathrm{s}}^{1 \gamma}(3 S \rightarrow 2 P)$. The direct two-photon decay and the two-step process of two-photon decays via the $2 S$ level are completely negligible in comparison.

\section{E. Generalizations of the EOMs}

Now we will turn to more general cases, accommodating points (i)-(iv) from Sec. II A for a more realistic description of the interaction process.

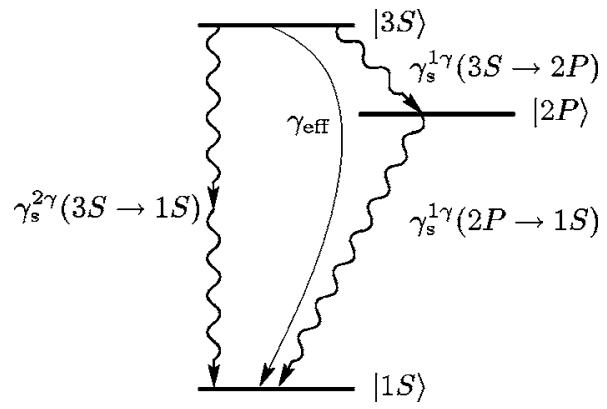

FIG. 9. Level scheme of the $3 S-1 S$ spontaneous decay. The twostep one-photon channel with the rates $\gamma_{s}^{1 \gamma}$ dominates over the direct two-photon decay, and can be expressed by a direct effective rate $\gamma_{\text {eff. }}$ The two-step two-photon channel via the $2 S$ state is omitted.

The light intensity in most experiments is not constant, e.g., the atom under consideration may move through an inhomogeneous laser profile. In this case, the laser intensity can be described in terms of the trajectory as $I(\mathbf{r}(t))$. The atom may also be excited with a pulsed laser field, where the time-dependent intensity $I(t)$ is known explicitly. In general, an analytic solution including the varying ac Stark effect and Rabi frequency is then either extremely convoluted or impossible to find. Nonetheless, the EOMs can then be readily integrated numerically, for example by using the RungeKutta routines from Ref. [33], if we include a timedependent intensity $I(t)$, specific to the experimental setup, into Eq. (10).

When we use the intensity in the context of two-photon spectroscopy, we must be aware of the resonator nature of most of these experiments. Typically the light is coupled into an enhancement cavity, mainly serving two purposes. First, the two photons necessary to drive the transition can be absorbed from opposite directions, leading to a cancellation of the first-order Doppler shift. Second, the total power circulating inside the interaction region is much larger than the in-coupling laser power, increasing the excitation probability.

If we consider a standing plane wave in the resonator on resonance, the intensity profile in the longitudinal direction is spatially modulated as

$$
I_{s}(x)=2 I \cos ^{2}(k x),
$$

where $I$ is the mean intensity, and where we take the cavity axis as the $x$ axis with $k$ being the modulus of the wave vector. If the atoms are fixed in space they simply observe the intensity at their respective position which ranges from 0 to $2 I$. In this case one faces the experimental problem of figuring out precisely where the atoms are, which could probably only be done for trapped ions.

For moving atoms in a standing wave, the description of a harmonic electric field with one frequency $\omega_{\mathrm{L}}$ is no longer appropriate, since in the frame of the atom, the electric field can only be described by a superposition of two oppositely running wave fields which are Doppler shifted with opposite signs, corresponding to the atomic velocity $v$ with respect to the laboratory frame. However, this superposition of electric fields, if inserted into the Hamiltonian (2), leads to the same 
set of equations (10), where $I$ is again the mean intensity, if the Doppler shift frequency is large compared to the characteristic frequencies occurring in the master equation (10):

$$
\omega_{\mathrm{L}}\left(\frac{1+\beta}{\sqrt{1-\beta^{2}}}-1\right) \gg \max \left\{\Omega, \gamma_{\mathrm{i}}, \gamma_{\mathrm{s}}\right\},
$$

where $\beta=v / c$. This is, in analogy with the rotating-wave approximation, a time-scale argument and it is not a strong restriction of the model presented here as the required minimum velocity to satisfy condition (19) for a thermal beam typically is quite low. For a Rabi frequency that occurs at some $\mathrm{MW} / \mathrm{m}^{2}$ in the hydrogen $1 S-2 S$ transition, this minimum velocity corresponds to a temperature on the order of $10^{-14} \mathrm{~K}$. From a laboratory frame point of view, condition (19) simply means that an atom passes through the intensity profile (18) quickly enough, such that it effectively averages over the nodes and antinodes of the field and can be treated as if it was driven by a homogeneous intensity $I$. An intensity averaging very similar to the movement of a single atom takes place when many atoms sample different fixed positions of the standing laser wave. Any slower variation of the intensity, in the sense that (19) is not satisfied, cannot be eliminated from the EOMs and has to be taken into account explicitly, such as the radial intensity variation of a Gaussian beam.

Consider a situation where the total electric field is a superposition of waves running in opposite directions and with different time-dependent intensities $I_{l}(t)$ and $I_{r}(t)$, as observed at the location of the atom, e.g., for excitation with counterpropagating pulses. For the description of the dynamics of the first-order Doppler free transition component, the following replacements have to be made in Eq. (10):

$$
\begin{gathered}
\Omega=2\left(2 \pi \beta_{g e}\right) I(t) \rightarrow \Omega=2\left(2 \pi \beta_{g e}\right) 2 \sqrt{I_{l}(t) I_{r}(t)}, \\
\Delta \nu_{\mathrm{ac}}=\beta_{\mathrm{ac}} I(t) \rightarrow \Delta \nu_{\mathrm{ac}}=\beta_{\mathrm{ac}}\left[I_{l}(t)+I_{r}(t)\right], \\
\gamma_{\mathrm{i}}=\left(2 \pi \beta_{\text {ioni }}\right) I(t) \rightarrow \gamma_{\mathrm{i}}=\left(2 \pi \beta_{\text {ioni }}\right)\left[I_{l}(t)+I_{r}(t)\right],
\end{gathered}
$$

again assuming that effective averaging over the maxima and minima of the standing field takes place, either because the atom is moving or by having many spatially distributed atoms contributing to the signal. The Rabi frequency in Eq. (20) scales with the product of the field amplitudes (i.e., field envelopes). To calculate the contribution of the Dopplershifted components, where the atom absorbs both photons from one beam only, the Rabi frequency becomes

$$
\Omega=2\left(2 \pi \beta_{g e}\right) I_{r}(t)
$$

for absorption of photons from the "right" beam, whereas the expressions for the ac Stark shift and the ionization rate are unaffected, since these processes are nonresonant. For these components, the first-order Doppler shift, including recoil effects, must be added to the laser detuning as given by Eq. (11).

Any additional spontaneous decay channels can also be fully included in a numerical integration algorithm. In addition to the intensity-dependent frequency shifts $\Delta \nu_{\text {ac }}$, any other frequency-shifting effect can be easily included into the numerical approach by adapting Eq. (11), as described already for the velocity-dependent second-order Doppler shift in Eq. (12).

Finally, in most cases, instead of a single atom one considers an ensemble of atoms, all of which may experience different intensities, Doppler shifts, and interaction times along different trajectories. The contribution of each single atom to the line shape can be calculated exactly like described above, and the line shape produced by the atomic ensemble is then the sum of all single-atom contributions, given that the single atoms interact independently with the laser. In a dilute beam experiment this condition is very well satisfied.

Together with the atomic constants presented in the following sections, the set of equations (10) are thus a tool for a wide range of problems in spectroscopy. For a specific implementation using a Monte Carlo method for the atomic ensemble in the case of the $1 S-2 S$ experiment, see [18].

Note that the transition matrix elements and Stark coefficients that enter into Eq. (10) vary slightly with the laser frequency. However, the detuning $\Delta \nu$ is typically a few kilohertz, whereas the optical resonance frequencies are on the order of $10^{15} \mathrm{~Hz}$. For the $1 S-2 S$ transition, the resulting relative variation of $\beta_{\mathrm{ac}}$ is on the order of $10^{-12}$, which is negligibly small. The question of different gauges, for two-photon excitation off resonance, has been discussed, e.g., in [34].

\section{CALCULATION OF TWO-PHOTON TRANSITION MATRIX ELEMENTS}

\section{A. Calculation}

In this and the next section, we will present the calculation of the two-photon transition matrix elements and the dynamic Stark coefficients, which are used in the quantum dynamics of the two-level system of Sec. II B.

In that section, the interacting system of atom and laser field has been described by an effective two-level Hamiltonian, focusing on the time-dependent atomic population of these levels. In order to describe transition matrix elements and dynamic Stark coefficients, involving virtual intermediate states, we have to consider the full, time-dependent Hamiltonian

$$
H(t)=H_{0}+V(t),
$$

where

$$
\begin{gathered}
H_{0}=\frac{\mathbf{p}^{2}}{2 m_{\mathrm{e}}}-\frac{Z e^{2}}{4 \pi \epsilon_{0} r}, \\
V(t)=V \frac{1}{2}\left[\exp \left(i \omega_{\mathrm{L}} t\right)+\exp \left(-i \omega_{\mathrm{L}} t\right)\right], \\
V=-e z \mathcal{E}_{\mathrm{L}},
\end{gathered}
$$

describing a one-electron system with nuclear charge number $Z$ in a harmonic laser field of angular frequency $\omega_{\mathrm{L}}$ and classical electric field amplitude $\mathcal{E}_{\mathrm{L}}$, linearly polarized in the $z$ direction. The electron mass is denoted by $m_{\mathrm{e}}$. The inter- 
action potential $V(t)$ is chosen to be in the length gauge, which has some advantages in the description of timedependent problems (for a detailed discussion see for example [35]), and the dipole approximation is made. The time-dependent two-photon transition matrix element connecting the ground state $|g\rangle$ and excited state $|e\rangle$ then reads

$$
\left\langle e\left|V(t) \frac{1}{\left(E_{g}+\hbar \omega_{\mathrm{L}}\right)-H_{0}} V(t)\right| g\right\rangle,
$$

where $E_{\phi}$ is the Schrödinger energy of any eigenstate $|\phi\rangle$ of $H_{0}$, depending only on its principal quantum number $n$ and is defined by

$$
E_{\phi}=-\frac{(Z \alpha)^{2} m_{\mathrm{e}} c^{2}}{2 n^{2}}
$$

with $\alpha$ being the fine-structure constant. Now we can establish the connection with the off-diagonal element of the twolevel Hamiltonian $\widetilde{H}$ from Sec. II B, Eq. (2), by equating

$$
\begin{array}{r}
-\left\langle e\left|V(t) \frac{1}{H_{0}-\left(E_{g}+\hbar \omega_{\mathrm{L}}\right)} V(t)\right| g\right\rangle \\
=\frac{\hbar \Omega}{2}\left[\exp \left(i \omega_{\mathrm{L}} t\right)+\exp \left(-i \omega_{\mathrm{L}} t\right)\right]^{2} .
\end{array}
$$

Observe that a minus sign is explicitly pulled out with respect to Eq. (26) in order to write the Green's function in the familiar form $1 /\left[H_{0}-E\right]$, with intermediate state energy $E$. With the definition of the two-photon Rabi-frequency from Eq. (6)

$$
\Omega=2\left(2 \pi \beta_{g e}\right) I,
$$

and the intensity of an electromagnetic plane wave

$$
I=\frac{1}{2} \epsilon_{0} c \mathcal{E}_{\mathrm{L}}^{2}
$$

we obtain for the time-independent two-photon transition matrix element

$$
\beta_{g e}=-\frac{e^{2}}{2 h c \epsilon_{0}}\left\langle e\left|z \frac{1}{H_{0}-\left(E_{g}+\hbar \omega_{\mathrm{L}}\right)} z\right| g\right\rangle .
$$

Note that the prefactor does not contain a factor $Z$, because it originates in the interaction part of the Hamiltonian (25c), describing the singly charged electron in the laser field.

In the literature, two common notations are used in expressing the transition matrix elements, and for completeness we will state that they are connected via the simple relation

$$
\begin{aligned}
& \left\langle e\left|z \frac{1}{\left(E_{g}+\hbar \omega_{\mathrm{L}}\right)-H_{0}} z\right| g\right\rangle \\
& \left.=\langle e| z \frac{1}{\left(E_{g}+\hbar \omega_{\mathrm{L}}\right)-H_{0}}\right]^{2}|g\rangle=\sum_{r} \frac{\langle e|z| r\rangle\langle r|z| g\rangle}{\left(E_{g}+\hbar \omega_{\mathrm{L}}\right)-E_{r}},
\end{aligned}
$$

with a complete set of $H_{0}$ eigenstates $|r\rangle$ denoted by

$$
1=\sum_{r}|r\rangle\langle r|
$$

The explicit sum over intermediate states at the bottom of Eq. (32) is widely used throughout the experimental laser spectroscopy literature, while the notation in the first line, involving the two-photon transition operator, is used mainly in publications inspired by a field-theoretic formalism (e.g., Refs. [36,37]). We will stick to the latter form in this paper, because it allows for an intuitive perturbation theoretic interpretation of (31) as the usual one-photon transition matrix element between the excited state $|e\rangle$ and the virtual intermediate state $|\delta g\rangle$ with energy $E_{g}+\hbar \omega_{\mathrm{L}}$. The intermediate state $|\delta g\rangle$ is apparently the first-order perturbation to the ground state $|g\rangle$, generated by the potential $V$, such that the matrix element itself is of second order in the perturbation potential.

At least three algorithms exist for the evaluation of matrix elements involving the nonrelativistic hydrogen Green's function as in Eq. (31). These are (i) a fully analytic evaluation based on the Sturmian representation of the radial Green's function for the hydrogen atom, in terms of Laguerre polynomials, (ii) a discretization of real space (of the radial variable) according to [38], and (iii) the solution of differential equations as, e.g., in [39]. We have used the first of these possibilities, the basic formalism of which has been laid out in [40-42], with the nonrelativistic hydrogen Green's function reading

$$
\left\langle\mathbf{r}_{1}\left|\frac{1}{H_{0}-E(\eta)}\right| \mathbf{r}_{2}\right\rangle=\sum_{l, m} g_{l}\left(r_{1}, r_{2} ; \eta\right) Y_{l m}\left(\theta_{1}, \varphi_{1}\right) Y_{l m}^{*}\left(\theta_{2}, \varphi_{2}\right),
$$

with

$$
\begin{aligned}
g_{l}\left(r_{1}, r_{2} ; \eta\right)= & \frac{2 m_{\mathrm{e}}}{\hbar^{2}}\left(\frac{2}{a_{0} \eta}\right)^{2 l+1}\left(r_{1} r_{2}\right)^{l} e^{-\left(r_{1}+r_{2}\right) / a_{0} \eta} \\
& \times \sum_{k=0}^{\infty} \frac{L_{k}^{2 l+1}\left(\frac{2 r_{1}}{a_{0} \eta}\right) L_{k}^{2 l+1}\left(\frac{2 r_{2}}{a_{0} \eta}\right)}{(k+1)_{2 l+1}(l+1+k-\eta)},
\end{aligned}
$$

where the usual spherical coordinates $\mathbf{r}_{i}=\left\{r_{i}, \theta_{i}, \phi_{i}\right\}$ are used, and $(a)_{n} \equiv \Gamma(a+n) / \Gamma(a)$ is the Pochhammer symbol. The Bohr radius is denoted by $a_{0}$, the symbols $L_{k}^{2 l+1}$ designate the associated Laguerre polynomials, and we employ the invertible energy parametrization

$$
\eta \equiv \eta(E)=\frac{Z \hbar}{a_{0}} \sqrt{-\frac{1}{2 m_{\mathrm{e}} E}}
$$

for conciseness of notation, converting any energy $E$ into the dimensionless parameter $\eta$, chosen such that for eigenstates $|\phi\rangle$ of $H_{0}$ with main quantum number $n$ we have $\eta\left(E_{\phi}\right)=n$. For the calculation of the transition matrix elements, we have to consider a virtual intermediate $P$ state in the propagator, with energy $E_{g}+\hbar \omega_{\mathrm{L}}$, and $\omega_{\mathrm{L}}$ is fixed by the two-photon resonance condition. Because the intermediate state is between two bound states, its energy is always negative, and therefore $\eta$ and the transition matrix elements are real. When we will come to the calculation of the dynamic Stark coeffi- 
cients in the next section, there will also be intermediate states in the continuum involved, and consequently the Stark coefficients will acquire an imaginary part.

Up to now, the states $|g\rangle$ and $|e\rangle$ have been characterized by the principal quantum number $n$ and orbital angular momentum quantum number $l$ only. Evaluated for any $S$-S transition, the two-photon transition operator

$$
T^{i j}=r^{i} \frac{1}{H_{0}-\left(E_{g}+\hbar \omega_{\mathrm{L}}\right)} r^{j}
$$

has isotropic symmetry, $\left\langle e\left|T^{x x}\right| g\right\rangle=\left\langle e\left|T^{y y}\right| g\right\rangle=\left\langle e\left|T^{z z}\right| g\right\rangle$, $\left\langle e\left|T^{i j}\right| g\right\rangle=0$ for $i \neq j$ and therefore transforms like a scalar under rotation. As a consequence, the two-photon transition matrix element for each individual allowed transition between fine structure (FS) and hyperfine structure (HFS) substrates can be obtained from $\beta_{g e}$ of the gross structure transition without any angular prefactors. We refer to transitions among states with different principal quantum number as the "gross structure" of the atom. For $S-D$ transitions, the rotational symmetry is broken by the $D$ state, and $\sum_{i}\left\langle e\left|T^{i i}\right| g\right\rangle=0$. We therefore calculate the reduced matrix elements of the rank-2 component $\beta_{g e}^{(2)}$ for orbital angular momentum eigenstates

$$
\beta_{g e}^{(2)}=-\frac{e^{2}}{2 h c \epsilon_{0}}\left\langle n^{\prime} D\|\mathbf{T}(2)\| n S\right\rangle,
$$

from which the transition matrix elements for specific magnetic sublevels can be obtained via the Wigner-Eckhart theorem. For the gross structure $S$ - $D$ transition the only transition which can be driven by linearly polarized light is the $m_{l}=0 \rightarrow m_{l}^{\prime}=0$ transition. For transitions between FS sublevels of angular momentum $J=L+S$ and $J^{\prime}=L^{\prime}+S$, where $S$ is the spin of the orbiting particle, the angular momenta have to be recoupled via the $6 j$ symbols [43]

$$
\begin{aligned}
\left\langle n^{\prime}\left(L^{\prime} S\right) J^{\prime}\|\mathbf{T}(2)\| n(L S) J\right\rangle & \\
= & \sqrt{(2 J+1)\left(2 J^{\prime}+1\right)} \times(-1)^{L^{\prime}+S+J+2}\left\{\begin{array}{ccc}
L^{\prime} & J^{\prime} & S \\
J & L & 2
\end{array}\right\} \\
& \times\left\langle n^{\prime} L^{\prime}\|\mathbf{T}(2)\| n L\right\rangle,
\end{aligned}
$$

because the two-photon transition operator only acts on the orbital angular momentum part of the wave function. If HFS sublevels are resolved, also the nuclear spin $I$ has to be taken into account in the same way. Denoting the total angular momenta by $F=J+I$ and $F^{\prime}=J^{\prime}+I$, one obtains

$$
\begin{aligned}
& \left\langle n^{\prime}\left(J^{\prime} I\right) F^{\prime}\|\mathbf{T}(2)\| n(J I) F\right\rangle \\
& =\sqrt{(2 F+1)\left(2 F^{\prime}+1\right)} \times(-1)^{J^{\prime}+I+F+2}\left\{\begin{array}{lll}
J^{\prime} & F^{\prime} & I \\
F & J & 2
\end{array}\right\} \\
& \quad \times\left\langle n^{\prime}\left(L^{\prime} S\right) J^{\prime}\|\mathbf{T}(2)\| n(L S) J\right\rangle .
\end{aligned}
$$

Finally, via the Wigner-Eckhart theorem, the transition matrix element between hyperfine magnetic sublevels $|g\rangle=\left|n S,(J I) F m_{F}\right\rangle$ and $|e\rangle=\left|n^{\prime} D,\left(J^{\prime} I\right) F^{\prime} m_{F}^{\prime}\right\rangle$ in a linearly $z$-polarized laser reads
TABLE II. Two-photon transition matrix elements $\beta_{g e}$ in units of $\mathrm{Hz}\left(\mathrm{W} / \mathrm{m}^{2}\right)^{-1}$ for $1 S \Leftrightarrow n S$ and $2 S \Leftrightarrow n S$ transitions, as defined in

\begin{tabular}{|c|c|c|}
\hline$n$ & $\begin{array}{c}1 S \Leftrightarrow n S \\
\beta_{g e} \\
{\left[\mathrm{~Hz}\left(\mathrm{~W} / \mathrm{m}^{2}\right)^{-1}\right]}\end{array}$ & $\begin{array}{c}2 S \Leftrightarrow n S \\
\beta_{g e} \\
{\left[\mathrm{~Hz}\left(\mathrm{~W} / \mathrm{m}^{2}\right)^{-1}\right]}\end{array}$ \\
\hline 2 & $3.68111 \times 10^{-5}$ & \\
\hline 3 & $1.00333 \times 10^{-5}$ & $1.23306 \times 10^{-3}$ \\
\hline 4 & $5.13409 \times 10^{-6}$ & $7.79393 \times 10^{-5}$ \\
\hline 5 & $3.28555 \times 10^{-6}$ & $-4.39666 \times 10^{-5}$ \\
\hline 6 & $2.35088 \times 10^{-6}$ & $-6.89568 \times 10^{-5}$ \\
\hline 7 & $1.79744 \times 10^{-6}$ & $-7.26216 \times 10^{-5}$ \\
\hline 8 & $1.43591 \times 10^{-6}$ & $-6.99362 \times 10^{-5}$ \\
\hline 9 & $1.18344 \times 10^{-6}$ & $-6.52683 \times 10^{-5}$ \\
\hline 10 & $9.98415 \times 10^{-7}$ & $-6.01620 \times 10^{-5}$ \\
\hline 11 & $8.57763 \times 10^{-7}$ & $-5.52069 \times 10^{-5}$ \\
\hline 12 & $7.47736 \times 10^{-7}$ & $-5.06214 \times 10^{-5}$ \\
\hline 13 & $6.59655 \times 10^{-7}$ & $-4.64686 \times 10^{-5}$ \\
\hline 14 & $5.87791 \times 10^{-7}$ & $-4.27450 \times 10^{-5}$ \\
\hline 15 & $5.28215 \times 10^{-7}$ & $-3.94201 \times 10^{-5}$ \\
\hline 16 & $4.78153 \times 10^{-7}$ & $-3.64542 \times 10^{-5}$ \\
\hline 17 & $4.35589 \times 10^{-7}$ & $-3.38061 \times 10^{-5}$ \\
\hline 18 & $3.99031 \times 10^{-7}$ & $-3.14375 \times 10^{-5}$ \\
\hline 19 & $3.67348 \times 10^{-7}$ & $-2.93136 \times 10^{-5}$ \\
\hline 20 & $3.39672 \times 10^{-7}$ & $-2.74039 \times 10^{-5}$ \\
\hline
\end{tabular}
Eq. (31), evaluated for atomic hydrogen $(Z=1)$, in the nonrelativistic dipole approximation. The electron mass is employed in the calculation; reduced-mass effects and the dependence of the results on the nuclear charge number $Z$ are given in Eq. (43). For transitions between $F=F^{\prime}=0$ and $F=F^{\prime}=1$ HFS sublevels, these values are valid with a unit angular prefactor and directly give the coefficient $\beta_{g e}$ defined in Eq. (41).

$$
\begin{aligned}
\beta_{g e}= & (-1)^{F^{\prime}-m_{F}^{\prime}}\left(\begin{array}{ccc}
F^{\prime} & 2 & F \\
-m_{F}^{\prime} & 0 & m_{F}
\end{array}\right) \\
& \times\left(-\frac{e^{2}}{2 h c \epsilon_{0}}\left\langle n^{\prime}\left(J^{\prime} I\right) F^{\prime}\|\mathbf{T}(2)\| n(J I) F\right\rangle\right),
\end{aligned}
$$

where the $3 j$ symbol is defined as in Ref. [43]. In summary, to arrive at $\beta_{g e}$ for a specific HFS transition, one starts with the value for $\beta_{g e}^{(2)}$ of the gross structure transition from Table III, solves Eq. (38) for the reduced matrix element, and sequentially inserts the results into Eqs. (39)-(41). For FS transitions, the step implied by Eq. (40) is skipped and in Eq. (41), one substitutes $F \rightarrow J, m_{F} \rightarrow m_{J}$ and $F^{\prime} \rightarrow J^{\prime}, m_{F}^{\prime} \rightarrow m_{J}^{\prime}$.

\section{B. Results for two-photon transitions}

Results for the two-photon transition matrix elements $\beta_{g e}$ for the transitions $1 S \Leftrightarrow n S(2 \leqslant n \leqslant 20)$ and $2 S \Leftrightarrow n S(3 \leqslant n$ $\leqslant 20)$ are given in Table II. For the transitions $1 S \Leftrightarrow n D$ and $2 S \Leftrightarrow n D(3 \leqslant n \leqslant 20)$, the reduced matrix elements for orbital angular momentum eigenstates are given in Table III. We have devoted Appendix E to the comparison with other 
TABLE III. Two-photon reduced transition matrix elements $\beta_{g e}^{(2)}$ in units of $\mathrm{Hz}\left(\mathrm{W} / \mathrm{m}^{2}\right)^{-1}$ for $1 S \Leftrightarrow n S$ and $2 S \Leftrightarrow n S$ transitions, as defined in Eq. (38), evaluated for atomic hydrogen $(Z=1)$, in the nonrelativistic dipole approximation. The electron mass is employed in the calculation; reduced-mass effects and the dependence of the results on the nuclear charge number $Z$ is given in Eq. (43). For specific transitions in FS and HFS sublevels, the angular prefactors in Eqs. (39)-(41) must be taken into account, as applicable.

\begin{tabular}{lcc}
\hline \hline & $1 S \Leftrightarrow n D$ & $2 S \Leftrightarrow n D$ \\
& $\beta_{g e}^{(2)}$ & $\beta_{g e}^{(2)}$ \\
$n$ & {$\left[\mathrm{~Hz}\left(\mathrm{~W} / \mathrm{m}^{2}\right)^{-1}\right]$} & {$\left[\mathrm{Hz}\left(\mathrm{W} / \mathrm{m}^{2}\right)^{-1}\right]$} \\
\hline 3 & $-6.16579 \times 10^{-5}$ & $4.23147 \times 10^{-4}$ \\
4 & $-3.89301 \times 10^{-5}$ & $-2.23806 \times 10^{-3}$ \\
5 & $-2.72644 \times 10^{-5}$ & $-1.75124 \times 10^{-3}$ \\
6 & $-2.04728 \times 10^{-5}$ & $-1.39563 \times 10^{-3}$ \\
7 & $-1.61138 \times 10^{-5}$ & $-1.15144 \times 10^{-3}$ \\
8 & $-1.31174 \times 10^{-5}$ & $-9.74048 \times 10^{-4}$ \\
9 & $-1.09516 \times 10^{-5}$ & $-8.39046 \times 10^{-4}$ \\
10 & $-9.32523 \times 10^{-6}$ & $-7.32816 \times 10^{-4}$ \\
11 & $-8.06659 \times 10^{-6}$ & $-6.47159 \times 10^{-4}$ \\
12 & $-7.06862 \times 10^{-6}$ & $-5.76793 \times 10^{-4}$ \\
13 & $-6.26133 \times 10^{-6}$ & $-5.18119 \times 10^{-4}$ \\
14 & $-5.59721 \times 10^{-6}$ & $-4.68586 \times 10^{-4}$ \\
15 & $-5.04300 \times 10^{-6}$ & $-4.26327 \times 10^{-4}$ \\
16 & $-4.57477 \times 10^{-6}$ & $-3.89943 \times 10^{-4}$ \\
17 & $-4.17491 \times 10^{-6}$ & $-3.58362 \times 10^{-4}$ \\
18 & $-3.83018 \times 10^{-6}$ & $-3.30751 \times 10^{-4}$ \\
19 & $-3.53048 \times 10^{-6}$ & $-3.06453 \times 10^{-4}$ \\
20 & $-3.26799 \times 10^{-6}$ & $-2.84944 \times 10^{-4}$ \\
\hline \hline
\end{tabular}

literature sources, where some of these results are also obtained, clarifying the prefactors and discussing some occasional inconsistencies, which we encountered in our literature search related to the problem. These nonrelativistic results are relevant for the given transitions in any bound two-body Coulomb system with nuclear charge number $Z$, where $1 \leqslant Z \lesssim 10$. Generally, we will refer to one of the particles involved as "the nucleus," although it need not be made up of baryons. Because the values, as listed in Tables II and III, have been obtained for $Z=1$ and infinite nuclear mass, the scaling with $Z$ and the dependence on the reduced mass of the system remain to be clarified. We use the scaling relations for the position operator as a function of $Z$ [32], and for the propagator denominators in (31). Note that the relevant laser frequency $\omega_{\mathrm{L}}(Z)=Z^{2} \omega_{\mathrm{L}}(Z=1)$ has to be scaled by a factor of $Z^{2}$ as compared to the corresponding frequency in hydrogen. Thus, we find that

$$
\beta_{g e}(Z)=\frac{1}{Z^{4}} \beta_{g e}(Z=1) .
$$

Consider a bound two-body system, consisting of particles with respective masses $m_{\mathrm{N}}$ for the nucleus and $m_{\mathrm{S}}$ for the other particle. This system is equivalent to a system with infinite nuclear mass and an orbiting particle with reduced mass $\mu$. It is therefore sufficient to replace the electron mass with the reduced mass in our calculations, bearing in mind that also the Bohr radius $a_{0}=4 \pi \epsilon_{0} \hbar^{2} / m_{\mathrm{e}} e^{2}$ needs to be replaced. Therefore, to obtain the transition matrix elements $\beta_{g e}$ for a two-body Coulomb system of nuclear charge number $Z$ and reduced mass $\mu$ (even for hydrogen itself), the values from Tables II and III must be multiplied by a factor

$$
\frac{1}{Z^{4}}\left(\frac{m_{\mathrm{e}}}{\mu}\right)^{3}=\frac{1}{Z^{4}}\left(\frac{m_{\mathrm{e}}\left(m_{\mathrm{N}}+m_{\mathrm{S}}\right)}{m_{\mathrm{N}} m_{\mathrm{S}}}\right)^{3} .
$$

This scaling law equally applies for the Stark coefficients $\beta_{\mathrm{ac}}$ and $\beta_{\text {ioni }}$ discussed below. Note that for atomic nuclei, we also obtain isotope shifts to the transition matrix elements via the dependence on the nuclear mass $m_{\mathrm{N}}$. In the next section, we will obtain nonrelativistic results for the dynamic Stark coefficients and consider radiative, relativistic, and field configuration corrections. We would like to mention here that these corrections, calculated for the transition matrix elements, are on the same order of magnitude as for the dynamic Stark coefficients.

\section{CALCULATION OF THE AC STARK SHIFT}

\section{A. Introduction}

This section treats the dynamic Stark shift of atomic energy levels, which is a consequence of the interaction with the laser field used to probe an atomic transition. This systematic shift cannot be suppressed experimentally and constitutes one major systematic effect in many precision spectroscopic experiments.

As opposed to one-photon resonant transitions, where the system of atom and laser field must be described in a nonperturbative dressed-state picture, in two-photon transitions involving the $1 S$ or $2 S$ states as ground states and an excited $S$ or $D$ state, the perturbation is always off resonant with respect to any one-photon transition. In contrast, a laser field driving the $3 S-5 S$ two-photon transition, is also one-photon resonant with the $5 S-15 P$ transition, according to Schrödinger theory [see Eq. (27)]. As a consequence, for our case of $1 S$ or $2 S$ states as ground states, the effect of the harmonic laser field as defined in Eq. (25) can be described in a perturbation theory approach for the present investigation. In the classical picture used for the electric field in this paper, the dynamic Stark shift falls into the domain of timedependent perturbation theory. However, in the more general theory, where the electromagnetic field is quantized, the same effect may be described in a time-independent framework with a static expression of the light field as a photon field with one macroscopically populated mode. The ac Stark shift has also been characterized as a stimulated radiative correction, because it results from a self-energy-like formalism when restricting the sum over virtual modes of the photon field to one single mode, the laser mode [44].

Both the classical time-dependent theory, and the timeindependent fully quantized treatment, in the limit of macroscopic photon number, yield the same physical result as presented in [45], where also the connection to the to the GellMann-Low-Sucher theorem has been reemphasized. In the 
notation of the previous section for the Hamiltonian [see Eqs. (24) and (25)] we can therefore express the dynamic Stark shift of the state $|\phi\rangle$ as [see Eq. (18) of [45]]

$$
\Delta E_{\mathrm{ac}}(\phi)=\frac{e^{2} \mathcal{E}_{\mathrm{L}}^{2}}{4} \sum_{ \pm}\left\langle\phi\left|z \frac{1}{E_{\phi}-H_{0} \pm \hbar \omega_{\mathrm{L}}} z\right| \phi\right\rangle .
$$

This form of $\Delta E_{\mathrm{ac}}(\phi)$ can be divided into a product, with one factor being the dynamic polarizability of the atom

$$
P_{\omega_{\mathrm{L}}}(\phi)=\sum_{ \pm}\left\langle\phi\left|z \frac{1}{H_{0}-E_{\phi} \pm \hbar \omega_{\mathrm{L}}} z\right| \phi\right\rangle,
$$

for an angular frequency $\omega_{\mathrm{L}}$, and a prefactor containing the laser intensity (30), such that we have

$$
\Delta E_{\mathrm{ac}}(\phi)=-\frac{e^{2}}{2 \epsilon_{0} c} I P_{\omega_{\mathrm{L}}}(\phi) .
$$

\section{B. Matrix elements}

The calculation of the dynamic Stark shift of any reference state $|\phi\rangle$ now reduces to calculating the matrix element of the dynamic polarizability (45), where we use the same analytic technique as for the two-photon transition matrix elements [see Eqs. (34)-(36)]. Note that the contributions of two intermediate states, with energies $E_{\phi}+\hbar \omega_{\mathrm{L}}$ and $E_{\phi}-\hbar \omega_{\mathrm{L}}$, have to be summed. These energies are determined by the choice of angular frequency of the laser field $\omega_{\mathrm{L}}=\left(E_{e}-E_{g}\right) / 2 \hbar$ in the two-photon resonant spectroscopy of the transition $g \Leftrightarrow e$. For laser detunings which drive the transition appreciably, the matrix elements are constant to a good approximation (see also end of Sec. II E). If the upper intermediate state is a continuum state, the energy parameter $\eta\left(E_{\phi}+\hbar \omega_{\mathrm{L}}>0\right)$ [see Eq. (36)] is complex, and the dynamic polarizability acquires an imaginary part, describing the population loss rate due to ionization. The real part of the ac Stark shift determines the frequency shift of the atomic level $|\phi\rangle$ in hertz via the relation

$$
\Delta \nu_{\mathrm{ac}}(\phi)=\frac{1}{h} \operatorname{Re}\left[\Delta E_{\mathrm{ac}}(\phi)\right]=\beta_{\mathrm{ac}}(\phi) I ;
$$

see also Eq. (5). The imaginary part of the ac Stark shift, if present, yields the decay constant of the probability amplitude of the atom to be in the reference state $|\phi\rangle$. The atomicstate population, as described by the diagonal elements of the density matrix in (10), is equal to the modulus squared of this probability amplitude, therefore the ionization rate of the population in $|\phi\rangle$ reads

$$
\gamma_{\mathrm{i}}=-\frac{2}{\hbar} \operatorname{Im}\left[\Delta E_{\mathrm{ac}}(\phi)\right]=2 \pi \beta_{\text {ioni }}(\phi) I,
$$

proportional to twice the imaginary part of the ac Stark shift. Note that we have given the ionization rate in units of angular frequency.

Standard selection rules for dipole transitions apply in determining the virtual intermediate states. In particular, as the laser is assumed to be linearly polarized in the $z$ direction, we can restrict the sum over $m$ of the intermediate states in the propagator (34) to the $m=0$ term. This choice of polarization does not restrict the generality of our discussion, because the initial $S$ states of the investigated transitions are spherically symmetric.

In Appendix A, one explicit polarizability matrix element $\left\langle 3 S\left|z\left(H_{0}-E\right)^{-1} z\right| 3 S\right\rangle$ is given. It involves the hypergeometric function ${ }_{2} F_{1}$, which originates from the infinite sum over Laguerre polynomials. In general, after the radial integrations we obtain a sum involving several hypergeometric functions, which can be reduced to a single one, using the contiguous relations for the hypergeometric functions (see, e.g., [46]). In Appendix C, the connection of $\beta_{\text {ioni }}$ to the usual ionization cross section is discussed.

To calculate the Stark shift coefficient $\beta_{\text {ac }}$ and ionization coefficient $\beta_{\text {ioni }}$ for FS and HFS states, the same considerations as for the transition operator apply. For $S$ states, the coefficients for the Schrödinger states are also applicable to each individual FS and HFS sublevel without modification. For an $n D$ state, the light shift operator

$$
Q^{i j}=\sum_{ \pm} r^{i} \frac{1}{H_{0}-E_{n} \pm \hbar \omega_{\mathrm{L}}} r^{j}
$$

consists of both a nonzero scalar component $\mathbf{Q}(0)$ and a rank-2 traceless component $\mathbf{Q}(2)$. To obtain the dynamic Stark shift coefficient $\beta_{\mathrm{ac}}(n D)$ in a linearly $z$-polarized laser field, the reduced matrix elements $\beta_{\mathrm{ac}}^{(0)}$ and $\beta_{\mathrm{ac}}^{(2)}$ have to be added after applying the appropriate angular prefactors.

In particular, the reduced matrix element $\beta_{\mathrm{ac}}^{(0)}$ needs no modification for FS and HFS sublevels, while $\beta_{\mathrm{ac}}^{(2)}$ must be multiplied by

$$
(-1)^{L+S+J}(2 J+1)\left\{\begin{array}{lll}
L & J & S \\
J & L & 2
\end{array}\right\}
$$

for a FS level with angular momentum $J=L+S$, where $L=2$, and additionally by

$$
(-1)^{J+I+F}(2 F+1)\left\{\begin{array}{lll}
J & F & I \\
F & J & 2
\end{array}\right\}
$$

if the state under consideration is a HFS level with angular momentum $F=J+I$. The Wigner-Eckhart theorem yields the dependence on the magnetic quantum number, such that, e.g., for a HFS state $|\phi\rangle=\left|n D,(J I) F m_{F}\right\rangle$ in a linearly $z$-polarized laser field, we obtain

$$
\begin{aligned}
\beta_{\mathrm{ac}}(\phi)= & \frac{1}{\sqrt{2 L+1}} \beta_{\mathrm{ac}}^{(0)}(n D)+(-1)^{L+S+2 J+I+2 F-m_{F}(2 J+1)} \\
& \times(2 F+1) \times\left(\begin{array}{ccc}
F & 2 & F \\
-m_{F} & 0 & m_{F}
\end{array}\right)\left\{\begin{array}{ccc}
L & J & S \\
J & L & 2
\end{array}\right\} \\
& \times\left\{\begin{array}{lll}
J & F & I \\
F & J & 2
\end{array}\right\} \beta_{\mathrm{ac}}^{(2)}(n D),
\end{aligned}
$$

where $L=2$ for $D$ states. The ionization coefficient $\beta_{\text {ioni }}$ of an $n D$ state is calculated in exactly the same way. 
TABLE IV. Dynamic Stark shift coefficients $\beta_{\text {ac }}$ and ionization coefficients $\beta_{\text {ioni }}$ for $1 S \Leftrightarrow n S$ transitions (on two-photon resonance), as defined in Eqs. (5), (9), (47), and (48), in the nonrelativistic dipole approximation, evaluated for nuclear charge number $Z=1$ and infinite nuclear mass. Reduced-mass effects and the dependence of the results on $Z$ can be included by multiplication with the scaling factor (43). For all $S$ states, the values are also applicable to all FS and HFS sublevels. The nonrelativistic treatment implies that the physical accuracy of the results given here is limited by corrections of relative order $(Z \alpha)^{2}$. Thus, about three decimals of the results in this table are relevant for a comparison of theory and experiment. Nevertheless, we indicate the data with a larger numerical accuracy, in order to facilitate the independent verification of the results.

\begin{tabular}{lccc}
\hline \hline $1 S \Leftrightarrow n S$ & $\beta_{\mathrm{ac}}(1 S)\left[\mathrm{Hz}\left(\mathrm{W} / \mathrm{m}^{2}\right)^{-1}\right]$ & $\beta_{\mathrm{ac}}(n S)\left[\mathrm{Hz}\left(\mathrm{W} / \mathrm{m}^{2}\right)^{-1}\right]$ & $\beta_{\text {ioni }}(n S)\left[\mathrm{Hz}\left(\mathrm{W} / \mathrm{m}^{2}\right)^{-1}\right]$ \\
\hline $1 S-2 S$ & $-2.67827 \times 10^{-5}$ & $1.39927 \times 10^{-4}$ & $1.20208 \times 10^{-4}$ \\
$1 S-3 S$ & $-3.02104 \times 10^{-5}$ & $9.80847 \times 10^{-5}$ & $2.02241 \times 10^{-5}$ \\
$1 S-4 S$ & $-3.18301 \times 10^{-5}$ & $8.66487 \times 10^{-5}$ & $7.10785 \times 10^{-6}$ \\
$1 S-5 S$ & $-3.26801 \times 10^{-5}$ & $8.20398 \times 10^{-5}$ & $3.35245 \times 10^{-6}$ \\
$1 S-6 S$ & $-3.31724 \times 10^{-5}$ & $7.97219 \times 10^{-5}$ & $1.85663 \times 10^{-6}$ \\
$1 S-7 S$ & $-3.34805 \times 10^{-5}$ & $7.83897 \times 10^{-5}$ & $1.13885 \times 10^{-6}$ \\
$1 S-8 S$ & $-3.36851 \times 10^{-5}$ & $7.75526 \times 10^{-5}$ & $7.50088 \times 10^{-7}$ \\
$1 S-9 S$ & $-3.38277 \times 10^{-5}$ & $7.69918 \times 10^{-5}$ & $5.20731 \times 10^{-7}$ \\
$1 S-10 S$ & $-3.39307 \times 10^{-5}$ & $7.65976 \times 10^{-5}$ & $3.76481 \times 10^{-7}$ \\
$1 S-11 S$ & $-3.40076 \times 10^{-5}$ & $7.63098 \times 10^{-5}$ & $2.81130 \times 10^{-7}$ \\
$1 S-12 S$ & $-3.40664 \times 10^{-5}$ & $7.60932 \times 10^{-5}$ & $2.15538 \times 10^{-7}$ \\
$1 S-13 S$ & $-3.41124 \times 10^{-5}$ & $7.59261 \times 10^{-5}$ & $1.68914 \times 10^{-7}$ \\
$1 S-14 S$ & $-3.41490 \times 10^{-5}$ & $7.57945 \times 10^{-5}$ & $1.34855 \times 10^{-7}$ \\
$1 S-15 S$ & $-3.41786 \times 10^{-5}$ & $7.56889 \times 10^{-5}$ & $1.09389 \times 10^{-7}$ \\
$1 S-16 S$ & $-3.42029 \times 10^{-5}$ & $7.56030 \times 10^{-5}$ & $8.99638 \times 10^{-8}$ \\
$1 S-17 S$ & $-3.42231 \times 10^{-5}$ & $7.55321 \times 10^{-5}$ & $7.48861 \times 10^{-8}$ \\
$1 S-18 S$ & $-3.42400 \times 10^{-5}$ & $7.54729 \times 10^{-5}$ & $6.30029 \times 10^{-8}$ \\
$1 S-19 S$ & $-3.42544 \times 10^{-5}$ & $7.54229 \times 10^{-5}$ & $5.35100 \times 10^{-8}$ \\
$1 S-20 S$ & $-3.42667 \times 10^{-5}$ & $7.53804 \times 10^{-5}$ & $4.58347 \times 10^{-8}$ \\
\hline \hline
\end{tabular}

\section{Results for $S-S$ transitions}

We have investigated the nonrelativistic dynamic Stark coefficients $\beta_{\text {ac }}$ and $\beta_{\text {ioni }}$ as defined in Eqs. (47) and (48), for laser frequencies on two-photon resonance with $S$-S and $S-D$ transitions. In particular, we give an overview of the values for transitions within the manifolds $1 S \Leftrightarrow n S$ in Table IV, $2 S \Leftrightarrow n S$ in Table V, $1 S \Leftrightarrow n D$ in Table VI, and $2 S \Leftrightarrow n D$ in Table VII up to $n=20$. The analytic results evaluated in the tables are obtained in the dipole approximation and with the approximation of retaining only the second-order in perturbation theory [see Eq. (44)]. In this case, both the frequency shift $\Delta \nu_{\mathrm{ac}}$ and the angular ionization rate $\gamma_{\mathrm{i}}$ are proportional to the light intensity. Corrections beyond the nonrelativistic dipole approach are discussed in the following Secs. IV D and IV E.

Some of the results listed in the tables have appeared in the literature before (e.g., Ref. [6]). Diverse unit systems and prefactors are encountered. Here, we present all results in SI units. An overview of the previous results is provided in Appendix E. Our results are in full agreement with those given in Refs. [5-7,10] and we add some more transitions to the analysis. In the comparison with results given in atomic units, as in [6], the conversion

$$
\beta_{\mathrm{ac}}(\phi)(\text { a.u. })=\beta_{\mathrm{ac}}(\phi)(\mathrm{SI}) \times \frac{\hbar^{2}}{m_{\mathrm{e}} a_{0}^{4} \alpha}
$$

has to be used, and analogously for $\beta_{\text {ioni }}$ [see also Eq. (E2) in Appendix E]. At this point we would like to reemphasize that in this section, the intensity is assumed to be constant at the location of the atom. For a discussion of the implications atoms moving in an inhomogeneous laser profile, see Sec. II B.

Concerning the dependence of the results on the nuclear charge number $Z$ and the reduced mass $\mu$ of the system, the same considerations apply as for the transition matrix elements in Sec. III. In particular, the values from Tables IV-VII must be multiplied by the scaling factor (43), for a specific two-body Coulomb system.

The $1 / Z^{4}$ dependence of the dynamic Stark coefficients, and especially the 16-fold reduction of the ionization coefficient, might be important in the context of planned measurements on trapped hydrogenlike helium. For spectroscopic experiments on systems with $Z>1$, the required light sources with ultrastable frequencies and sufficient intensity have recently been demonstrated to be within reach in the near future [15]. 
TABLE V. Dynamic Stark shift coefficients and ionization coefficients (on two-photon resonance) as in Table IV, but for $2 S \Leftrightarrow n S$ transitions.

\begin{tabular}{lccc}
\hline \hline $2 S \Leftrightarrow n S$ & $\beta_{\mathrm{ac}}(2 S)\left[\mathrm{Hz}\left(\mathrm{W} / \mathrm{m}^{2}\right)^{-1}\right]$ & $\beta_{\mathrm{ac}}(n S)\left[\mathrm{Hz}\left(\mathrm{W} / \mathrm{m}^{2}\right)^{-1}\right]$ & $\beta_{\text {ioni }}(n S)\left[\mathrm{Hz}\left(\mathrm{W} / \mathrm{m}^{2}\right)^{-1}\right]$ \\
\hline $2 S-3 S$ & $-7.18795 \times 10^{-4}$ & $-6.99895 \times 10^{-3}$ & 0 \\
$2 S-4 S$ & $-9.47799 \times 10^{-4}$ & $2.11716 \times 10^{-3}$ & $1.25626 \times 10^{-3}$ \\
$2 S-5 S$ & $-1.16885 \times 10^{-3}$ & $1.70310 \times 10^{-3}$ & $4.65485 \times 10^{-4}$ \\
$2 S-6 S$ & $-1.36379 \times 10^{-3}$ & $1.52064 \times 10^{-3}$ & $2.28478 \times 10^{-4}$ \\
$2 S-7 S$ & $-1.52869 \times 10^{-3}$ & $1.42368 \times 10^{-3}$ & $1.30721 \times 10^{-4}$ \\
$2 S-8 S$ & $-1.66537 \times 10^{-3}$ & $1.36562 \times 10^{-3}$ & $8.23925 \times 10^{-5}$ \\
$2 S-9 S$ & $-1.77773 \times 10^{-3}$ & $1.32791 \times 10^{-3}$ & $5.55288 \times 10^{-5}$ \\
$2 S-10 S$ & $-1.86994 \times 10^{-3}$ & $1.30194 \times 10^{-3}$ & $3.93145 \times 10^{-5}$ \\
$2 S-11 S$ & $-1.94583 \times 10^{-3}$ & $1.28325 \times 10^{-3}$ & $2.89099 \times 10^{-5}$ \\
$2 S-12 S$ & $-2.00858 \times 10^{-3}$ & $1.26932 \times 10^{-3}$ & $2.19090 \times 10^{-5}$ \\
$2 S-13 S$ & $-2.06078 \times 10^{-3}$ & $1.25865 \times 10^{-3}$ & $1.70162 \times 10^{-5}$ \\
$2 S-14 S$ & $-2.10451 \times 10^{-3}$ & $1.25029 \times 10^{-3}$ & $1.34890 \times 10^{-5}$ \\
$2 S-15 S$ & $-2.14138 \times 10^{-3}$ & $1.24361 \times 10^{-3}$ & $1.08794 \times 10^{-5}$ \\
$2 S-16 S$ & $-2.17269 \times 10^{-3}$ & $1.23819 \times 10^{-3}$ & $8.90578 \times 10^{-6}$ \\
$2 S-17 S$ & $-2.19945 \times 10^{-3}$ & $1.23372 \times 10^{-3}$ & $7.38465 \times 10^{-6}$ \\
$2 S-18 S$ & $-2.22247 \times 10^{-3}$ & $1.23000 \times 10^{-3}$ & $6.19281 \times 10^{-6}$ \\
$2 S-19 S$ & $-2.24238 \times 10^{-3}$ & $1.22687 \times 10^{-3}$ & $5.24540 \times 10^{-6}$ \\
$2 S-20 S$ & $-2.25971 \times 10^{-3}$ & $1.22420 \times 10^{-3}$ & $4.48258 \times 10^{-6}$ \\
\hline \hline
\end{tabular}

The analytic calculation of the matrix elements for the highly excited states would be a formidable task without the use of computer algebra software [47]. The reason that the calculations are carried out up to rather high quantum numbers is that the availability of a large number of results permits the evaluation of the asymptotic limit of the light shifts

TABLE VI. Dynamic Stark coefficients as in Table IV, but for $1 S \Leftrightarrow n D$ transitions. The respective coefficients for the $1 S$ state, $\beta_{\mathrm{ac}}(1 S)$, are identical with those from Table IV, and are therefore not listed again. For FS and HFS sublevels of an $n D$ state, the appropriate angular prefactors from Eqs. (50)-(52) must be taken into account.

\begin{tabular}{lcccc}
\hline \hline & $\begin{array}{c}\beta_{\mathrm{ac}}^{(0)}(n D) \\
{\left[\mathrm{Hz}\left(\mathrm{W} / \mathrm{m}^{2}\right)^{-1}\right]}\end{array}$ & $\begin{array}{c}\beta_{\mathrm{ac}}^{(2)}(n D) \\
{\left[\mathrm{Hz}\left(\mathrm{W} / \mathrm{m}^{2}\right)^{-1}\right]}\end{array}$ & $\begin{array}{c}\beta_{\text {ioni }}^{(0)}(n D) \\
{\left[\mathrm{Hz}\left(\mathrm{W} / \mathrm{m}^{2}\right)^{-1}\right]}\end{array}$ & $\begin{array}{c}\beta_{\text {ioni }}^{(2)}(n D) \\
{\left[\mathrm{Hz}\left(\mathrm{W} / \mathrm{m}^{2}\right)^{-1}\right]}\end{array}$ \\
\hline $1 S \Leftrightarrow n D$ & $2.11378 \times 10^{-4}$ & $1.30662 \times 10^{-5}$ & $3.67432 \times 10^{-6}$ & $-2.11508 \times 10^{-6}$ \\
$1 S-4 D$ & $1.90315 \times 10^{-4}$ & $4.70825 \times 10^{-6}$ & $1.39210 \times 10^{-6}$ & $-8.11183 \times 10^{-7}$ \\
$1 S-5 D$ & $1.81694 \times 10^{-4}$ & $2.24738 \times 10^{-6}$ & $6.70000 \times 10^{-7}$ & $-3.92504 \times 10^{-7}$ \\
$1 S-6 D$ & $1.77255 \times 10^{-4}$ & $1.25298 \times 10^{-6}$ & $3.74221 \times 10^{-7}$ & $-2.19848 \times 10^{-7}$ \\
$1 S-7 D$ & $1.74652 \times 10^{-4}$ & $7.71734 \times 10^{-7}$ & $2.30562 \times 10^{-7}$ & $-1.35678 \times 10^{-7}$ \\
$1 S-8 D$ & $1.72990 \times 10^{-4}$ & $5.09668 \times 10^{-7}$ & $1.52256 \times 10^{-7}$ & $-8.96938 \times 10^{-8}$ \\
$1 S-9 D$ & $1.71863 \times 10^{-4}$ & $3.54487 \times 10^{-7}$ & $1.05879 \times 10^{-7}$ & $-6.24189 \times 10^{-8}$ \\
$1 S-10 D$ & $1.71062 \times 10^{-4}$ & $2.56634 \times 10^{-7}$ & $7.66377 \times 10^{-8}$ & $-4.52039 \times 10^{-8}$ \\
$1 S-11 D$ & $1.70472 \times 10^{-4}$ & $1.91828 \times 10^{-7}$ & $5.72754 \times 10^{-8}$ & $-3.37962 \times 10^{-8}$ \\
$1 S-12 D$ & $1.70025 \times 10^{-4}$ & $1.47183 \times 10^{-7}$ & $4.39391 \times 10^{-8}$ & $-2.59345 \times 10^{-8}$ \\
$1 S-13 D$ & $1.69678 \times 10^{-4}$ & $1.15414 \times 10^{-7}$ & $3.44509 \times 10^{-8}$ & $-2.03388 \times 10^{-8}$ \\
$1 S-14 D$ & $1.69403 \times 10^{-4}$ & $9.21865 \times 10^{-8}$ & $2.75145 \times 10^{-8}$ & $-1.62467 \times 10^{-8}$ \\
$1 S-15 D$ & $1.69182 \times 10^{-4}$ & $7.48066 \times 10^{-8}$ & $2.23252 \times 10^{-8}$ & $-1.31844 \times 10^{-8}$ \\
$1 S-16 D$ & $1.69001 \times 10^{-4}$ & $6.15416 \times 10^{-8}$ & $1.83651 \times 10^{-8}$ & $-1.08470 \times 10^{-8}$ \\
$1 S-17 D$ & $1.68851 \times 10^{-4}$ & $5.12407 \times 10^{-8}$ & $1.52901 \times 10^{-8}$ & $-9.03172 \times 10^{-9}$ \\
$1 S-18 D$ & $1.68726 \times 10^{-4}$ & $4.31191 \times 10^{-8}$ & $1.28659 \times 10^{-8}$ & $-7.60040 \times 10^{-9}$ \\
$1 S-19 D$ & $1.68620 \times 10^{-4}$ & $3.66289 \times 10^{-8}$ & $1.09289 \times 10^{-8}$ & $-6.45656 \times 10^{-9}$ \\
$1 S-20 D$ & $1.68529 \times 10^{-4}$ & $3.13799 \times 10^{-8}$ & $9.36238 \times 10^{-9}$ & $-5.53143 \times 10^{-9}$ \\
\hline \hline
\end{tabular}


TABLE VII. Dynamic Stark coefficients as in Table VI, but for $2 S \Leftrightarrow n D$ transitions. The respective coefficients for the $2 S$ state, $\beta_{\mathrm{ac}}(2 S)$, are identical with those from Table V.

\begin{tabular}{lcccc}
\hline \hline & $\begin{array}{c}\beta_{\mathrm{ac}}^{(0)}(n D) \\
{\left[\mathrm{Hz}\left(\mathrm{W} / \mathrm{m}^{2}\right)^{-1}\right]}\end{array}$ & $\begin{array}{c}\beta_{\mathrm{ac}}^{(2)}(n D) \\
{\left[\mathrm{Hz}\left(\mathrm{W} / \mathrm{m}^{2}\right)^{-1}\right]}\end{array}$ & $\begin{array}{c}\beta_{\mathrm{ioni}}^{(0)}(n D) \\
{\left[\mathrm{Hz}\left(\mathrm{W} / \mathrm{m}^{2}\right)^{-1}\right]}\end{array}$ & $\begin{array}{c}\beta_{\mathrm{ioni}}^{(2)}(n D) \\
{\left[\mathrm{Hz}\left(\mathrm{W} / \mathrm{m}^{2}\right)^{-1}\right]}\end{array}$ \\
\hline $2 S \Leftrightarrow n D$ & $-1.17698 \times 10^{-2}$ & $4.99866 \times 10^{-3}$ & 0 & 0 \\
$2 S-4 D$ & $5.47527 \times 10^{-3}$ & $-1.64045 \times 10^{-4}$ & $1.91609 \times 10^{-3}$ & $-1.10511 \times 10^{-3}$ \\
$2 S-5 D$ & $3.91342 \times 10^{-3}$ & $1.89977 \times 10^{-4}$ & $5.84726 \times 10^{-4}$ & $-3.43343 \times 10^{-4}$ \\
$2 S-6 D$ & $3.40856 \times 10^{-3}$ & $1.64090 \times 10^{-4}$ & $2.62740 \times 10^{-4}$ & $-1.55670 \times 10^{-4}$ \\
$2 S-7 D$ & $3.17241 \times 10^{-3}$ & $1.21898 \times 10^{-4}$ & $1.43176 \times 10^{-4}$ & $-8.52682 \times 10^{-5}$ \\
$2 S-8 D$ & $3.03968 \times 10^{-3}$ & $8.99553 \times 10^{-5}$ & $8.75750 \times 10^{-5}$ & $-5.23245 \times 10^{-5}$ \\
$2 S-9 D$ & $2.95642 \times 10^{-3}$ & $6.74381 \times 10^{-5}$ & $5.78591 \times 10^{-5}$ & $-3.46451 \times 10^{-5}$ \\
$2 S-10 D$ & $2.90022 \times 10^{-3}$ & $5.15428 \times 10^{-5}$ & $4.03987 \times 10^{-5}$ & $-2.42271 \times 10^{-5}$ \\
$2 S-11 D$ & $2.86026 \times 10^{-3}$ & $4.01348 \times 10^{-5}$ & $2.94079 \times 10^{-5}$ & $-1.76557 \times 10^{-5}$ \\
$2 S-12 D$ & $2.83068 \times 10^{-3}$ & $3.17867 \times 10^{-5}$ & $2.21175 \times 10^{-5}$ & $-1.32900 \times 10^{-5}$ \\
$2 S-13 D$ & $2.80812 \times 10^{-3}$ & $2.55609 \times 10^{-5}$ & $1.70777 \times 10^{-5}$ & $-1.02684 \times 10^{-5}$ \\
$2 S-14 D$ & $2.79046 \times 10^{-3}$ & $2.08355 \times 10^{-5}$ & $1.34753 \times 10^{-5}$ & $-8.10656 \times 10^{-6}$ \\
$2 S-15 D$ & $2.77637 \times 10^{-3}$ & $1.71910 \times 10^{-5}$ & $1.08282 \times 10^{-5}$ & $-6.51678 \times 10^{-6}$ \\
$2 S-16 D$ & $2.76493 \times 10^{-3}$ & $1.43390 \times 10^{-5}$ & $8.83715 \times 10^{-6}$ & $-5.32030 \times 10^{-6}$ \\
$2 S-17 D$ & $2.75549 \times 10^{-3}$ & $1.20777 \times 10^{-5}$ & $7.30950 \times 10^{-6}$ & $-4.40183 \times 10^{-6}$ \\
$2 S-18 D$ & $2.74762 \times 10^{-3}$ & $1.02634 \times 10^{-5}$ & $6.11704 \times 10^{-6}$ & $-3.68459 \times 10^{-6}$ \\
$2 S-19 D$ & $2.74098 \times 10^{-3}$ & $8.79168 \times 10^{-6}$ & $5.17212 \times 10^{-6}$ & $-3.11603 \times 10^{-6}$ \\
$2 S-20 D$ & $2.73533 \times 10^{-3}$ & $7.58614 \times 10^{-6}$ & $4.41334 \times 10^{-6}$ & $-2.65934 \times 10^{-6}$ \\
\hline \hline & & & & \\
\hline
\end{tabular}

for $n \rightarrow \infty$. Thus, if theoretical predictions are sought for higher $n$ than those included in the tables, these can be obtained by fits involving inverse powers of the principal quantum number to the real and to the imaginary parts of the data in Tables IV and V; see also Table Ic in [6]. The limit as $n \rightarrow \infty$ might be interesting especially in view of the long lifetime of highly excited $S$ states, which allows for a high spectral resolution [48].

\section{Relativistic and radiative corrections}

The transition matrix elements and dynamic Stark coefficients involved in the quantum dynamics (10) have been calculated nonrelativistically, which is a good approximation for low- $Z$ systems. For use in high-precision measurements, however, one should at least consider leading-order relativistic and radiative corrections to ensure the validity of the approximation.

For an estimation of magnitude, it is helpful to remark that the leading relativistic corrections are of relative order $(Z \alpha)^{2}$, whereas the leading radiative corrections are of order $\alpha(Z \alpha)^{2} \ln \left[(Z \alpha)^{-2}\right]$. It follows that for atomic hydrogen, they do not exceed a relative magnitude of $10^{-4}$, and therefore at least three digits in the Tables IV and $\mathrm{V}$ are significant regarding a comparison of theory and experiment in hydrogen and low- $Z$ hydrogenlike systems.

In the electric dipole approximation, the relativistic corrections to the dynamic polarizability of $1 S$ and $2 S$ have recently been calculated to all orders in $Z \alpha$ in the form of generalized hypergeometric series [39], employing the fully relativistic Dirac-Coulomb propagator. Regarding an experimental verification of the relativistic, multipole, and retarda- tion corrections, as well as the radiative contributions, we only mention that the required accurate measurement of the laser intensity would be the most difficult task.

In this section, we only give results for the mentioned corrections to the dynamic polarizability for selected states, which are of interest in the spectroscopic analysis at hand. The purpose is to demonstrate that these corrections are below the currently accessible experimental precision.

We compare the radiative corrections to the dynamic polarizability $\delta P_{\omega_{\mathrm{L}}}(\phi)$ in leading logarithmic order to the uncorrected matrix element $P_{\omega_{\mathrm{L}}}(\phi)$ defined in Eq. (45), where the real and imaginary parts are treated separately to reflect the correction to the dynamic Stark coefficient $\delta \beta_{\mathrm{ac}}(\phi)$ and the correction to the ionization coefficient $\delta \beta_{\text {ioni }}(\phi)$ :

$$
\begin{gathered}
\eta_{\mathrm{rad}} R_{\mathrm{rad}}(\phi)=\frac{\operatorname{Re}\left[\delta P_{\omega_{\mathrm{L}}}(\phi)\right]}{\operatorname{Re}\left[P_{\omega_{\mathrm{L}}}(\phi)\right]}=\frac{\delta \beta_{\mathrm{ac}}(\phi)}{\beta_{\mathrm{ac}}(\phi)}, \\
\eta_{\mathrm{rad}} I_{\mathrm{rad}}(\phi)=\frac{\operatorname{Im}\left[\delta P_{\omega_{\mathrm{L}}}(\phi)\right]}{\operatorname{Im}\left[P_{\omega_{\mathrm{L}}}(\phi)\right]}=\frac{\delta \beta_{\text {ioni }}(\phi)}{\beta_{\text {ioni }}(\phi)},
\end{gathered}
$$

with $\eta_{\mathrm{rad}}=\alpha(Z \alpha)^{2} \ln \left[(Z \alpha)^{-2}\right]$. For the relativistic corrections, the definitions of $R_{\text {rel }}(\phi)$ and $I_{\text {rel }}(\phi)$ are completely analogous, but with $\eta_{\text {rel }}=(Z \alpha)^{2}$.

For the transitions $1 S-2 S, 1 S-3 S$, and $2 S-3 S$ the relative magnitude of the radiative corrections is given in Table VIII.

The relativistic corrections are not defined unambiguously, because of the existence of field-configurationdependent corrections which enter at the same order of magnitude $(Z \alpha)^{2}$ (see Sec. IV E). In addition, we have neglected 
TABLE VIII. Radiative corrections to the dynamic polarizability in leading logarithmic order as defined in Eqs. (54) and (55).

\begin{tabular}{lccc}
\hline \hline $\begin{array}{l}\text { Transition } \\
|g\rangle \Leftrightarrow|e\rangle\end{array}$ & $R_{\mathrm{rad}}(g)$ & $R_{\mathrm{rad}}(e)$ & $I_{\mathrm{rad}}(e)$ \\
\hline $1 S-2 S$ & 3.617 & $5.281 \times 10^{-1}$ & $-8.077 \times 10^{-1}$ \\
$1 S-3 S$ & 4.045 & $1.042 \times 10^{-1}$ & -1.103 \\
$2 S-3 S$ & 8.861 & $2.937 \times 10^{+1}$ & \\
\hline \hline
\end{tabular}

corrections due to the interaction of the magnetic field with the electron. In contrast, the leading radiative correction is well defined because it entails the electric-dipole approximation. The relativistic corrections considered include the contributions from the zitterbewegung of the electron, the spinorbit coupling, and the leading-order relativistic momentum correction, and are given in Table IX, again for the transitions $1 S-2 S, 1 S-3 S$, and $2 S-3 S$.

\section{E. Field-configuration-dependent corrections}

In this section we will consider the leading field configuration correction to the dynamic polarizability, which is due to the fact that the laser field, interacting with an atom of finite extension, is not strictly a dipole field, but also varies in space for a fixed time $t$. We will consider the case of a plane standing wave, which approximates the situation in the hydrogen $1 S-2 S$ experiment [16] well enough for the discussion of this correction. For other field configurations like, e.g., tight foci, the results of this section (see Table $\mathrm{X}$ ) are not applicable, but must be reevaluated to suit the corresponding geometry. For this reason we give a rather detailed description of the calculation of the corrections here.

Up to now, all results were obtained in the electric dipole approximation, where the interaction part of the Hamiltonian was expressed as

$$
V_{\text {dip }}(t)=-e \mathbf{r} \cdot \mathbf{E}(t)
$$

In long-wavelength QED (see [37] and Eq. (3.59) of [49]), the interaction with an electric field varying in both space and time can be written as

TABLE IX. Relativistic corrections to the dynamic polarizability as defined in analogy to Eqs. (54) and (55).

\begin{tabular}{lccc}
\hline \hline $\begin{array}{l}\text { Transition } \\
|g\rangle \Leftrightarrow|e\rangle\end{array}$ & $R_{\text {rel }}(g)$ & $R_{\text {rel }}(e)$ & $I_{\text {rel }}(e)$ \\
\hline $1 S-2 S$ & & & \\
$1 S-3 S$ & -1.185 & $-1.903 \times 10^{-1}$ & $2.047 \times 10^{-1}$ \\
$2 S-3 S$ & -1.280 & $-7.410 \times 10^{-2}$ & $1.199 \times 10^{-1}$ \\
\hline \hline
\end{tabular}

TABLE X. Field configuration corrections to the dynamic polarizability occurring in a plane standing wave, as defined in Eqs. (64) and (65).

\begin{tabular}{lccc}
\hline \hline $\begin{array}{l}\text { Transition } \\
|g\rangle \Leftrightarrow|e\rangle\end{array}$ & $R_{\mathrm{fc}}(g)$ & $R_{\mathrm{fc}}(e)$ & $I_{\mathrm{fc}}(e)$ \\
\hline $1 S-2 S$ & $-2.256 \times 10^{-2}$ & $-1.988 \times 10^{-1}$ & $3.750 \times 10^{-2}$ \\
$1 S-3 S$ & $-3.244 \times 10^{-2}$ & -1.105 & $1.185 \times 10^{-1}$ \\
$2 S-3 S$ & $-1.008 \times 10^{-2}$ & $3.771 \times 10^{-3}$ & \\
\hline \hline
\end{tabular}

$$
\begin{aligned}
V_{\mathrm{LW}}(t, \mathbf{r})= & -e \mathbf{r} \cdot \mathbf{E}(t, \mathbf{0})-\left.\frac{e}{2} r^{i} r^{j} \frac{\partial E^{i}(t, \mathbf{r})}{\partial r^{j}}\right|_{\mathbf{r}=\mathbf{0}} \\
& -\left.\frac{e}{6} r^{i} r^{j} r^{k} \frac{\partial^{2} E^{i}(t, \mathbf{r})}{\partial r^{j} \partial r^{k}}\right|_{\mathbf{r}=\mathbf{0}} .
\end{aligned}
$$

For a plane standing wave of linearly $z$-polarized light with wave vector $\mathbf{k}$, aligned along the $x$ direction, the electric field is

$$
\mathbf{E}(t, x)=\hat{e}_{z} \mathcal{E}_{\mathrm{L}} \cos (\omega t) \cos (k x),
$$

and consequently for the last term in (57) we obtain simply

$$
V_{\mathrm{LW}}^{(2)}=\frac{e}{6} z k^{2} x^{2} \mathcal{E}_{\mathrm{L}} \cos (\omega t)
$$

The leading-order correction to the dynamic polarizability of state $|\phi\rangle$ therefore reads

$$
\delta P_{\omega_{\mathrm{L}}}(\phi)=-\frac{k^{2}}{3} \sum_{ \pm}\left\langle\phi\left|z \frac{1}{H_{0}-E_{\phi} \pm \hbar \omega_{\mathrm{L}}} x^{2} z\right| \phi\right\rangle,
$$

because the lower-order contributions vanish for symmetry reasons:

$$
\begin{aligned}
& -\frac{k}{2}\left\langle\phi\left|z \frac{1}{H_{0}-E} x z\right| \phi\right\rangle=0, \\
& \frac{k^{2}}{4}\left\langle\phi\left|z x \frac{1}{H_{0}-E} x z\right| \phi\right\rangle=0 .
\end{aligned}
$$

The modulus of the wave vector $k$ of the standing wave is determined by the two-photon resonance condition

$$
k=\frac{3}{16}(Z \alpha)^{2} \frac{c m_{\mathrm{e}}}{\hbar}=\frac{\omega_{\mathrm{L}}}{c}
$$

for the $1 S-2 S$ transition, which also yields the frequency $\omega_{\mathrm{L}}$ at which the polarizability itself is evaluated. With these parameters fixed, we obtain the leading-order correction, relative to the polarizability in dipole approximation

$$
\eta_{\mathrm{fc}} R_{\mathrm{fc}}(\phi)=\frac{\operatorname{Re}\left[\delta P_{\omega_{\mathrm{L}}}(\phi)\right]}{\operatorname{Re}\left[P_{\omega_{\mathrm{L}}}(\phi)\right]},
$$




$$
\eta_{\mathrm{fc}} I_{\mathrm{fc}}(\phi)=\frac{\operatorname{Im}\left[\delta P_{\omega_{\mathrm{L}}}(\phi)\right]}{\operatorname{Im}\left[P_{\omega_{\mathrm{L}}}(\phi)\right]},
$$

where again the corrections to the real part $R_{\mathrm{fc}}$ and to the imaginary part $I_{\mathrm{fc}}$ are considered separately, and the factor

$$
\eta_{\mathrm{fc}}=(Z \alpha)^{2}
$$

is used. We see that the field-configuration-dependent corrections are of the same order in $Z \alpha$ as the relativistic corrections. Note that the correction $\delta P_{\omega_{\mathrm{L}}}(\phi)$ is of the order $(Z \alpha)^{-2}$, while the polarizability $P_{\omega_{\mathrm{L}}}(\phi)$ is of the order $(Z \alpha)^{-4}$. The results, evaluated for the same transitions as in the previous subsection, are listed in Table X.

\section{CONCLUSIONS}

In this paper, we have presented in a unified treatment the quantum dynamics and atomic constants describing the twophoton excitation process in ongoing and planned precision experiments on bound two-body Coulomb systems, including the dependence on $Z$ and the reduced mass of the system.

The influence on the linewidth by ionization from the excited atomic state has been studied in the density matrix formalism, and an analytic solution to the corresponding equations of motion has been discussed, in which features like spectral hole burning due to the ionization have been observed (see Figs. 1-4). Ionization is therefore a decisive feature of the quantum dynamics and cannot be ignored in high-precision two-photon spectroscopy experiments.

In particular, we have focused on the $1 S-2 S$ transition in hydrogen and hydrogenlike helium. In the latter case, we observed that the effective loss rate of the atomic population is significantly smaller than expected, if one considers the ionization coefficient of the excited state without taking into account the excitation dynamics.

Potential applications include detection schemes in planned experiments on hydrogen and $\mathrm{He}^{+}$.
In this paper we have restricted the discussion to the case of monochromatic cw excitation of a single atom, initially in the ground state. In realistic cases, the effect of an inhomogeneous laser intensity profile and the velocity distributions of an atomic ensemble can be taken into account with a numerical integration of the equations of motion presented here. Two such applications can be found in [18], on the analysis of the hydrogen $1 S-2 S$ transition, and in [50], discussing high-precision spectroscopy in hydrogenlike helium.

Finally, results of relativistic, radiative, and fieldconfiguration-dependent corrections to the dynamic polarizability have been briefly discussed for the most interesting transitions in the context of this paper. These corrections, which have some theoretical interest of their own, are found to be small, as expected, but might become relevant in the future.

\section{ACKNOWLEDGMENTS}

The authors appreciate helpful conversations with V. Yakhontov, J. Evers, M. Macovei, P. J. Mohr, and R. Moshammer. U.D.J. gratefully acknowledges support by the Deutsche Forschungsgemeinschaft (DFG) via the Heisenberg program. N.K. acknowledges support by the DFG (Grant No. 436RUS113/769/0-1), the RFBR (Grants No. 03-02-04029, and No. 04-0217443) and the Alexander von Humboldt Stiftung.

\section{APPENDIX A: $3 S$ DYNAMIC POLARIZABILITY}

Below, we give as an example the exact analytic solution for a matrix element $\left\langle\phi\left|z\left\{1 /\left[H_{0}-E(\eta)\right]\right\} z\right| \phi\right\rangle$, the reference state being the $|3 S\rangle$ state. The energy parametrization $E(\eta)$ is defined in Eq. (36). The Bohr radius is denoted by $a_{0}, m_{\mathrm{e}}$ is the electron mass, and $H_{0}$ is the unperturbed hydrogen Hamiltonian (25a):

$$
\begin{aligned}
\left\langle 3 S\left|z \frac{1}{H_{0}-E(\eta)} z\right| 3 S\right\rangle= & \frac{m_{\mathrm{e}} a_{0}^{4}}{Z^{4} \hbar^{2}} \times \frac{54 \eta^{2}}{\left(9-\eta^{2}\right)^{8}}\left[110008287-87156324 \eta^{2}+29819745 \eta^{4}-6022998 \eta^{6}-1259712 \eta^{7}\right. \\
& +2106081 \eta^{8}+1912896 \eta^{9}-504792 \eta^{10}-737856 \eta^{11}-31041 \eta^{12}+84672 \eta^{13}+15538 \eta^{14} \\
& \left.+3456 \eta^{7}\left(27-7 \eta^{2}\right)^{2}\left(1-\eta^{2}\right)_{2} F_{1}\left(1,-\eta, 1-\eta,\left(\frac{3-\eta}{3+\eta}\right)^{2}\right)\right]
\end{aligned}
$$

For higher excited states, the analytic results have a more complex analytic structure, which necessitates the use of computer algebra systems [47]. In Ref. [36], the authors have observed that the ${ }_{2} F_{1}$ hypergeometric function occurs in the result for integrals containing the Schrödinger-Coulomb Green's function in the above form.

\section{APPENDIX B: ANALYTIC SOLUTION FOR THE POPULATION}

In this section, we give the explicit expression for the time-dependent line shape of excited atoms $\rho_{e e}^{\prime}(\Delta \omega, t)$ and the ground-state population $\rho_{g g}^{\prime}(\Delta \omega, t)$, satisfying the master 
equation (10). The ionization probability can be obtained by evaluating $1-\rho_{e e}^{\prime}(\Delta \omega, t)-\rho_{g g}^{\prime}(\Delta \omega, t)$, which is needed to model the line shape in an experiment using the detection of ionized particles instead of intact atoms in the excited state. We obtain

$$
\begin{aligned}
\rho_{e e}^{\prime}(\Delta \omega, t)= & C \Omega^{2}\left\{e^{-\Omega_{c} t} \Omega_{b}\left[\Omega_{a}\left(\Omega_{a}^{2}-\Omega_{b}^{2}\right) \cosh \left(\Omega_{a} t\right)+\Omega_{c}\left(3 \Omega_{a}^{2}+\Omega_{b}^{2}-4 \Omega_{c}^{2}\right) \sinh \left(\Omega_{a} t\right)\right]+e^{\Omega_{c} t} \Omega_{a}\left[\Omega_{b}\left(\Omega_{b}^{2}-\Omega_{a}^{2}\right) \cosh \left(\Omega_{b} t\right)\right.\right. \\
& \left.\left.-\Omega_{c}\left(\Omega_{a}^{2}+3 \Omega_{b}^{2}-4 \Omega_{c}^{2}\right) \sinh \left(\Omega_{b} t\right)\right]\right\}
\end{aligned}
$$

$$
\begin{aligned}
\rho_{g g}^{\prime}(\Delta \omega, t)= & \rho_{e e}^{\prime}(\Delta \omega, t)+C \times\left[2 e ^ { - \Omega _ { c } t } \Omega _ { b } \left(\Omega _ { a } \left[8 \Omega_{c}^{4}+\left(\Omega_{a}^{2}-\Omega_{b}^{2}\right)\left(\Delta \omega^{2}+\Omega_{a}^{2}\right)+\Omega_{c}\left(\gamma_{\mathrm{i}}+\gamma_{\mathrm{s}}\right)\left(2 \Delta \omega^{2}+\Omega_{a}^{2}+\Omega_{b}^{2}-2 \Omega_{c}^{2}\right)\right.\right.\right. \\
& \left.-\Omega_{c}^{2}\left(5 \Omega_{a}^{2}+3 \Omega_{b}^{2}\right)\right] \cosh \left(\Omega_{a} t\right)+\left\{\Omega_{c}\left[\Omega_{a}^{2}\left(\Omega_{a}^{2}+3 \Omega_{b}^{2}\right)+\Omega_{c}^{2}\left(\Omega_{b}^{2}-\Omega_{a}^{2}-4 \Omega_{c}^{2}\right)+\Delta \omega^{2}\left(3 \Omega_{a}^{2}+\Omega_{b}^{2}-4 \Omega_{c}^{2}\right)\right]+\frac{\gamma_{\mathrm{i}}+\gamma_{\mathrm{s}}}{2}\right. \\
& \left.\left.\times\left[\left(\Omega_{a}^{2}-\Omega_{b}^{2}+4 \Omega_{c}^{2}\right)\left(\Delta \omega^{2}+\Omega_{a}^{2}+\Omega_{c}^{2}\right)-8 \Omega_{a}^{2} \Omega_{c}^{2}\right]\right\} \sinh \left(\Omega_{a} t\right)\right)+2 e_{c} \Omega_{a}\left(\Omega _ { b } \left[8 \Omega_{c}^{4}+\left(\Omega_{b}^{2}-\Omega_{a}^{2}\right)\left(\Delta \omega^{2}+\Omega_{b}^{2}\right)\right.\right. \\
& \left.-\Omega_{c}\left(\gamma_{\mathrm{i}}+\gamma_{\mathrm{s}}\right)\left(2 \Delta \omega^{2}+\Omega_{a}^{2}+\Omega_{b}^{2}-2 \Omega_{c}^{2}\right)-\Omega_{c}^{2}\left(3 \Omega_{a}^{2}+5 \Omega_{b}^{2}\right)\right] \cosh \left(\Omega_{b} t\right)-\left\{\Omega _ { c } \left[\Omega_{b}^{2}\left(\Omega_{b}^{2}+3 \Omega_{a}^{2}\right)+\Omega_{c}^{2}\left(\Omega_{a}^{2}-\Omega_{b}^{2}-4 \Omega_{c}^{2}\right)\right.\right. \\
& \left.\left.\left.\left.+\Delta \omega^{2}\left(\Omega_{a}^{2}+3 \Omega_{b}^{2}-4 \Omega_{c}^{2}\right)\right]-\frac{\gamma_{\mathrm{i}}+\gamma_{\mathrm{s}}}{2}\left[\left(\Omega_{b}^{2}-\Omega_{a}^{2}+4 \Omega_{c}^{2}\right)\left(\Delta \omega^{2}+\Omega_{b}^{2}+\Omega_{c}^{2}\right)-8 \Omega_{b}^{2} \Omega_{c}^{2}\right]\right\} \sinh \left(\Omega_{b} t\right)\right)\right]
\end{aligned}
$$

with

$$
\begin{aligned}
& C=\frac{e^{-\left(\gamma_{\mathrm{i}}+\gamma_{\mathrm{s}}\right) t / 2}}{2 \Omega_{a} \Omega_{b}\left[\left(\Omega_{a}-\Omega_{b}\right)^{2}-4 \Omega_{c}^{2}\right]\left[\left(\Omega_{a}+\Omega_{b}\right)^{2}-4 \Omega_{c}^{2}\right]}, \\
& \Omega_{a}=\frac{\sqrt{2\left(\gamma_{\mathrm{i}}+\gamma_{\mathrm{s}}\right)^{2}-8 \Delta \omega^{2}-8 \Omega^{2}-3 \gamma_{\mathrm{s}} \Omega^{2} / \Omega_{c}-D-K}}{2 \sqrt{6}}, \\
& \Omega_{b}=\frac{\sqrt{2\left(\gamma_{\mathrm{i}}+\gamma_{\mathrm{s}}\right)^{2}-8 \Delta \omega^{2}-8 \Omega^{2}+3 \gamma_{\mathrm{s}} \Omega^{2} / \Omega_{c}-D-K}}{2 \sqrt{6}}, \\
& \Omega_{c}=\frac{\sqrt{\left(\gamma_{\mathrm{i}}+\gamma_{\mathrm{s}}\right)^{2}-4 \Delta \omega^{2}-4 \Omega^{2}+D+K}}{2 \sqrt{6}}, \\
& K=\frac{16 \Delta \omega^{4}+\left[\left(\gamma_{\mathrm{i}}+\gamma_{\mathrm{s}}\right)^{2}-4 \Omega^{2}\right]^{2}+8 \Delta \omega^{2}\left[4 \Omega^{2}-7\left(\gamma_{\mathrm{i}}+\gamma_{\mathrm{s}}\right)^{2}\right]}{4 D} \\
& D=2^{2 / 3}\left\{27 \gamma_{\mathrm{i}} \Omega^{2}\left(\gamma_{\mathrm{i}}+\gamma_{\mathrm{s}}\right)^{3}+2\left(\frac{5}{4}\left(\gamma_{\mathrm{i}}+\gamma_{\mathrm{s}}\right)^{2}+\Delta \omega^{2}+\Omega^{2}\right)^{3}-\frac{9}{2} \gamma_{\mathrm{i}} \Omega^{2}\left(\gamma_{\mathrm{i}}+\gamma_{\mathrm{s}}\right)\left[5\left(\gamma_{\mathrm{i}}+\gamma_{\mathrm{s}}\right)^{2}+4 \Delta \omega^{2}+4 \Omega^{2}\right]-\frac{9}{8}\left(\gamma_{\mathrm{i}}+\gamma_{\mathrm{s}}\right)\right. \\
& \times\left[4 \Delta \omega^{2}+5\left(\gamma_{i}+\gamma_{s}\right)^{2}+4 \Omega^{2}\right]\left[4 \Delta \omega^{2}\left(\gamma_{i}+\gamma_{s}\right)+\left(\gamma_{i}+\gamma_{s}\right)^{3}+2\left(2 \gamma_{i}+\gamma_{s}\right) \Omega^{2}\right]+\frac{27}{16}\left[4 \Delta \omega^{2}\left(\gamma_{i}+\gamma_{s}\right)+\left(\gamma_{i}+\gamma_{s}\right)^{3}\right. \\
& \left.+2 \Omega^{2}\left(2 \gamma_{\mathrm{i}}+\gamma_{\mathrm{s}}\right)\right]^{2}+\left[-4\left(\Delta \omega^{4}+\frac{1}{16}\left[\left(\gamma_{\mathrm{i}}+\gamma_{\mathrm{s}}\right)^{2}-4 \Omega^{2}\right]^{2}+\frac{1}{2} \Delta \omega^{2}\left[-7\left(\gamma_{\mathrm{i}}+\gamma_{\mathrm{s}}\right)^{2}+4 \Omega^{2}\right]\right)^{3}+\frac{1}{1024}\left\{\left(\gamma_{\mathrm{i}}+\gamma_{\mathrm{s}}\right)^{6}-64 \Delta \omega^{6}-64 \Omega^{6}\right.\right. \\
& -12 \Omega^{2}\left(\gamma_{\mathrm{i}}+\gamma_{\mathrm{s}}\right)^{4}+24 \Omega^{4}\left(2 \gamma_{\mathrm{i}}^{2}+4 \gamma_{\mathrm{i}} \gamma_{\mathrm{s}}-7 \gamma_{\mathrm{s}}^{2}\right)-48 \Delta \omega^{4}\left[11\left(\gamma_{\mathrm{i}}+\gamma_{\mathrm{s}}\right)^{2}+4 \Omega^{2}\right]+12 \Delta \omega^{2}\left[11\left(\gamma_{\mathrm{i}}+\gamma_{\mathrm{s}}\right)^{4}-40 \Omega^{2}\left(\gamma_{\mathrm{i}}+\gamma_{\mathrm{s}}\right)^{2}\right. \\
& \left.\left.\left.\left.-16 \Omega^{4}\right]\right\}^{2}\right\}^{1 / 2}\right\}^{1 / 3}
\end{aligned}
$$


In this form, the auxiliary variables $\Omega_{a}, \Omega_{b}, \Omega_{c}, K$, and $D$ are not necessarily real, depending on the relative magnitude of the spontaneous decay rate $\gamma_{\mathrm{s}}$, the ionization rate $\gamma_{\mathrm{i}}$, and the two-photon Rabi frequency $\Omega$. Nevertheless, the resulting populations $\rho_{e e}$ and $\rho_{g g}$ are real quantities.

In earlier works dealing with multiphoton ionization (see, e.g., $[51,52])$, where the above solution is contained in the limiting case $\gamma_{\mathrm{s}}=0$ [see Eqs. (13)], the emphasis is on efficient ionization, and in strong fields, either the spontaneous decay of the intermediate state is neglected in comparison with the ionization rate or, if present in the master equations, it is treated numerically.

However, in experiments that detect the excited-state line shape, and are therefore carried out in a relatively weak field, the spontaneous decay rate and the ionization rate can be of comparable magnitude; therefore only the above solution (B1) describes the constant-intensity case appropriately, as detailed in Sec. II D. Of course, a more compact expression would be highly desirable; however, we were not able to simplify it further. Quite generally, spontaneous decay channels complicate the exact solution of the dynamics, because it reduces the symmetry of the EOMs, as has been shown for a different set of master equations, considering autoionizing states in a laser field $[53,54]$.

\section{APPENDIX C: CALCULATION OF THE PHOTOIONIZATION CROSS SECTION}

The photoionization cross sections obtained in this section are generally applicable to the regime of ionization by lowintensity laser radiation with a minimum photon energy of $Z^{2} \times 13.6 \mathrm{eV} / n^{2}$, where $n=1,2$ is the principal quantum number of the initial atomic state and $Z$ is the nuclear charge. The theory for this process has been very well understood since the 1930s [4], including the $Z$ scaling, and a comparison of the results constitutes an independent verification of the ionization rate coefficients $\beta_{\text {ioni }}$ obtained in Sec. IV.

In analogy to bound states (27), one can assign to the continuum state a generalized principal quantum number $n^{\prime}$, which is real and positive, such that the energy of the electron in the continuum can be written as

$$
E_{n^{\prime}}=-\frac{(Z \alpha)^{2} m_{\mathrm{e}} c^{2}}{2\left(i^{\prime}\right)^{2}}>0 .
$$

Here, the continuum threshold is taken as the zero point of the energy scale, such that bound states extend into the negative energy region (this is different from the conventions used in an early article [4] on this subject).

If we consider a dipole transition from the bound $2 S$ state to the continuum $\varepsilon P$ state, the one-photon ionization cross section of a single atom for linearly polarized light is proportional to the square of the transition matrix element and reads

$$
\sigma_{2 S}=\frac{\pi e^{2} \omega_{\mathrm{L}}}{\epsilon_{0} c}|\langle 2 S|z| \varepsilon P\rangle|^{2},
$$

where $\omega_{\mathrm{L}}$ is the angular frequency of the incident laser radiation, and $e$ is the electron charge. The radial integrals encountered can be calculated in at least two ways: (i) direct integration of terms generated by the series representation of the ${ }_{1} F_{1}$ hypergeometric function occurring in the $\varepsilon P$ wave function, and (ii) contour integration using an integral representation of the hypergeometric function (see, e.g., [32]) and evaluation of the residues. Both (i) and (ii) lead to the same result

$$
|\langle 2 S|z| \varepsilon P\rangle|^{2}=\frac{2^{17} a_{0}^{4} m_{\mathrm{e}}}{3 Z^{4} \hbar^{2}} \frac{\exp \left[-4 n^{\prime} \operatorname{arccot}\left(n^{\prime} / 2\right)\right]}{1-\exp \left(-2 \pi n^{\prime}\right)} \frac{n^{\prime 10}\left(1+n^{\prime 2}\right)}{\left(4+n^{\prime 2}\right)^{6}} .
$$

Observe that the left-hand side is proportional to $1 / Z^{4}$ because the matrix element contains one continuum state. For two bound states, the transition matrix element squared would scale as $1 / Z^{2}$. In [55], one can find a result differing by a factor of $\frac{3}{2}$ from Eq. (C3).

For the case of an incident laser angular frequency of one-half of the $1 S-2 S$ transition frequency, the generalized quantum number of the continuum state is $n^{\prime}=2 \sqrt{2}$, and we obtain the nonrelativistic result

$$
\sigma_{2 S}=\frac{1}{Z^{2}} 6.174 \times 10^{-18} \mathrm{~cm}^{2} .
$$

We can compare the ionization rate coefficient $\beta_{\text {ioni }}$ from Sec. IV directly with the $2 S$ cross section calculated above using the relation

$$
\sigma_{\text {ioni }}=2 \pi \beta_{\text {ioni }} \hbar \omega_{\mathrm{L}}=\frac{1}{Z^{2}} 6.174 \times 10^{-18} \mathrm{~cm}^{2},
$$

which agrees with (C4). Here, the $Z$ scaling of the ionization cross section is the result of a factor $Z^{-4}$ for the ionization coefficient $\beta_{\text {ioni }}$ and the $Z^{2}$ scaling of the photon energy $\hbar \omega_{\mathrm{L}}$, required for maintaining two-photon resonance with a given transition. In Table XI the ionization cross sections in $\mathrm{cm}^{2}$ for a set of excited $n S$ levels is given, as obtained from the imaginary part of the dynamic Stark coefficient $\beta_{\text {ioni }}$. All considerations concerning the relativistic and radiative corrections to $\beta_{\text {ioni }}$ given in Sec. IV D also apply to the ionization cross section through relation (C5). Their magnitude is small and below the current precision of measurement, decreasing even for larger nuclear charge. This behavior is different than in most radiative corrections of nondynamic processes [56].

\section{APPENDIX D: COMBINED INDUCED-SPONTANEOUS TWO-PHOTON DECAY}

In the context of laser driven two-photon $S$-S transitions and two-photon spontaneous emission, one also has to consider the two-step process of combined induced-spontaneous two-photon decay of the excited level. In this process, the interaction of the excited atom with the laser field and the vacuum modes leads to a final state of the system, where the atom is in the ground state, the laser mode photon number is increased or decreased by 1 , and one photon is spontaneously emitted into an empty mode of the electromagnetic field. One contribution is depicted Fig. 10, where the interaction with the laser field takes place before the spontaneous emission. 
TABLE XI. Ionization cross sections for hydrogen atoms $(Z=1)$ in the excited state $n S$, at an incident laser frequency on two-photon resonance with the respective $1 S \Leftrightarrow n S$ or $2 S \Leftrightarrow n S$ transition, as obtained via Eq. (C5) from the dynamic Stark coefficient $\beta_{\text {ioni }}$. This table is provided for convenient comparison with other cross section calculations. Therefore, the values are here given in the usual units $\mathrm{cm}^{2}$ instead of SI units.

\begin{tabular}{lcc}
\hline \hline$n$ & $1 S \Leftrightarrow n S$ & $2 S \Leftrightarrow n S$ \\
& $\sigma_{\text {ioni }}(n S)\left(\mathrm{cm}^{2}\right)$ & $\sigma_{\text {ioni }}(n S)\left(\mathrm{cm}^{2}\right)$ \\
\hline 2 & $6.174 \times 10^{-18}$ & \\
3 & $1.231 \times 10^{-18}$ & 0 \\
4 & $4.563 \times 10^{-19}$ & $1.613 \times 10^{-17}$ \\
5 & $2.204 \times 10^{-19}$ & $6.694 \times 10^{-18}$ \\
6 & $1.236 \times 10^{-19}$ & $3.477 \times 10^{-18}$ \\
7 & $7.640 \times 10^{-20}$ & $2.055 \times 10^{-18}$ \\
8 & $5.057 \times 10^{-20}$ & $1.322 \times 10^{-18}$ \\
9 & $3.522 \times 10^{-20}$ & $9.037 \times 10^{-19}$ \\
10 & $2.552 \times 10^{-20}$ & $6.462 \times 10^{-19}$ \\
11 & $1.909 \times 10^{-20}$ & $4.786 \times 10^{-19}$ \\
12 & $1.466 \times 10^{-20}$ & $3.647 \times 10^{-19}$ \\
13 & $1.150 \times 10^{-20}$ & $2.844 \times 10^{-19}$ \\
14 & $9.188 \times 10^{-21}$ & $2.262 \times 10^{-19}$ \\
15 & $7.458 \times 10^{-21}$ & $1.830 \times 10^{-19}$ \\
16 & $6.137 \times 10^{-21}$ & $1.501 \times 10^{-19}$ \\
17 & $5.111 \times 10^{-21}$ & $1.247 \times 10^{-19}$ \\
18 & $4.301 \times 10^{-21}$ & $1.047 \times 10^{-19}$ \\
19 & $3.654 \times 10^{-21}$ & $8.881 \times 10^{-20}$ \\
20 & $3.131 \times 10^{-21}$ & $7.598 \times 10^{-20}$ \\
\hline \hline
\end{tabular}

We describe the interaction of the atom with the laser mode with the second-quantized interaction Hamiltonian

$$
H_{\mathrm{L}}=-e z \sqrt{\frac{\hbar \omega_{\mathrm{L}}}{2 \epsilon_{0} \mathcal{V}}}\left(a_{\mathrm{L}}^{\dagger}+a_{\mathrm{L}}\right)
$$

where the dipole approximation is used and $a_{\mathrm{L}}$ and $a_{\mathrm{L}}^{\dagger}$ are the annihilation and creation operators for a photon of energy

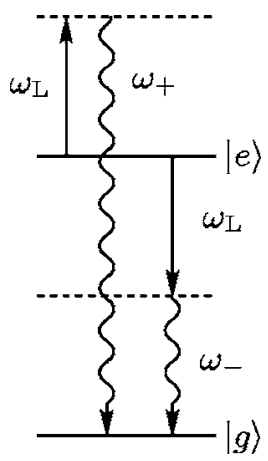

FIG. 10. Combined induced-spontaneous decay process leading to a depopulation of the excited state $|e\rangle$. Absorption or stimulated emission of one laser photon with energy $\hbar \omega_{\mathrm{L}}$ and subsequent spontaneous decay of the virtual intermediate states (dashed) to the ground state can take place, via emission of a photon of energy $\hbar \omega_{+}$ or $\hbar \omega_{-}$, respectively.
TABLE XII. Combined induced-spontaneous two-photon decay coefficients $\beta_{\text {is }}$ between two $S$ states interacting with a laser field on two-photon resonance, as defined in Eq. (D6).

\begin{tabular}{lc}
\hline \hline Transition & $\beta_{\text {is }}\left[\mathrm{Hz} /\left(\mathrm{W} / \mathrm{m}^{2}\right)\right]$ \\
\hline $1 S-2 S$ & $8.05160 \times 10^{-11} Z^{-2}$ \\
$1 S-3 S$ & $1.11235 \times 10^{-11} Z^{-2}$ \\
$2 S-3 S$ & $1.00370 \times 10^{-8} Z^{-2}$ \\
\hline \hline
\end{tabular}

$\hbar \omega_{\mathrm{L}}$ in the laser mode, given the quantization volume $\mathcal{V}$. The interaction with the vacuum modes is described by the interaction Hamiltonian

$$
H_{\mathrm{V}}=-e \sum_{\{\mathbf{k}, \lambda\} \neq \mathrm{L}} \mathbf{r} \cdot \boldsymbol{\epsilon}_{\lambda}(\mathbf{k}) \sqrt{\frac{\hbar \omega_{k}}{2 \epsilon_{0} \mathcal{V}}}\left[a_{\lambda}^{\dagger}(\mathbf{k})+a_{\lambda}(\mathbf{k})\right] .
$$

Here, $\mathbf{k}$ is the wave vector of any mode of the electromagnetic field except the laser mode, with polarization vector $\boldsymbol{\epsilon}_{\lambda}(\mathbf{k})$. We denote the initial state by $\left|\phi_{0}\right\rangle=\left|e, n_{\mathrm{L}}, 0\right\rangle$, with $n_{\mathrm{L}}$ photons in the laser mode and no photons in any other mode. To evaluate the transition rate of the combined inducedspontaneous decay, one needs to evaluate the imaginary part of the principal term of the fourth-order energy perturbation

$$
\begin{aligned}
& \Delta E_{\mathrm{LV}}^{(4)}\left(\phi_{0}\right) \\
& \quad=\left\langle\phi_{0}\left|H_{\mathrm{LV}} \frac{1}{E_{0}-H_{0}} H_{\mathrm{LV}} \frac{1}{E_{0}-H_{0}} H_{\mathrm{LV}} \frac{1}{E_{0}-H_{0}} H_{\mathrm{LV}}\right| \phi_{0}\right\rangle,
\end{aligned}
$$

where $H_{\mathrm{LV}}=H_{\mathrm{L}}+H_{\mathrm{V}}$, taking into account only those terms that contain both the laser interaction and the vacuum interaction in second order. Here, the unperturbed Hamiltonian $H_{0}$ includes the atomic spectrum as well as the field modes:

$$
H_{0}=\sum_{n} E_{n}|n\rangle\langle n|+\hbar \omega_{\mathrm{L}} a_{\mathrm{L}}^{\dagger} a_{\mathrm{L}}+\sum_{\{\mathbf{k}, \lambda\} \neq \mathrm{L}} \hbar \omega_{k} a_{\lambda}^{\dagger}(\mathbf{k}) a_{\lambda}(\mathbf{k}) .
$$

From the resonances caused by the possible final states $\left|g, n_{\mathrm{L}}+1,1_{z}\left(\mathbf{k}_{-}\right)\right\rangle$and $\left|g, n_{\mathrm{L}}-1,1_{z}\left(\mathbf{k}_{+}\right)\right\rangle$, we obtain the induced-spontaneous decay rate $\gamma_{\text {is }}$ as

$$
\begin{gathered}
\gamma_{\mathrm{is}}=\left(2 \pi \beta_{\mathrm{is}}\right) I \\
\beta_{\mathrm{is}}=\frac{4 e^{4}}{6 \pi c^{4} \epsilon_{0}^{2} h}\left(\omega_{-}^{3}\left|\left\langle e\left|z \frac{1}{H_{\mathrm{A}}-\left(E_{e}-\hbar \omega_{\mathrm{L}}\right)} z\right| g\right\rangle\right|^{2}\right. \\
+\omega_{+}^{3} \mid\left\langle\left. e\left|z \frac{1}{H_{\mathrm{A}}-\left(E_{e}+\hbar \omega_{\mathrm{L}}\right)} z\right| g\right|^{2}\right)
\end{gathered}
$$

where $H_{\mathrm{A}}=\sum_{n} E_{n}|n\rangle\langle n|$ is the atomic part of the unperturbed Hamiltonian, and $\omega_{ \pm}=c\left|\mathbf{k}_{ \pm}\right|=\omega_{e g} \pm \omega_{\mathrm{L}}$. For a small set of transitions, we list the results in Table XII. It is obvious that for typical intensities of several $\mathrm{MW} / \mathrm{m}^{2}$, as considered in Sec. II, the contribution of this process to the width of the excited state is small, when compared to ionization and spontaneous decay. 


\section{APPENDIX E: OTHER LITERATURE SOURCES}

\section{1. $1 S-2 S$ transition}

This section is dedicated to a comparison of the results given in this paper with previous work. For the convenience of the reader, we try to describe all the prefactors in detail, where different conventions and different units are used. The collection of articles covered in the following is not claimed to be comprehensive.

For the $1 S-2 S$ transition matrix element, we obtain $\beta_{g e}=3.68111 \times 10^{-5} \mathrm{~Hz} /\left(\mathrm{W} / \mathrm{m}^{2}\right)$ (see Table II), with

$$
\begin{aligned}
\beta_{g e} & =-\frac{e^{2}}{2 h c \epsilon_{0}}\left\langle 2 S\left|z \frac{1}{H_{0}-\left(E_{1 S}+\hbar \omega_{\mathrm{L}}\right)} z\right| 1 S\right\rangle \\
& =-\frac{e^{2}}{2 h c \epsilon_{0}} \sum_{n} \frac{\langle 2 S|z| n\rangle\langle n|z| 1 S\rangle}{E_{n}-E_{1 S}-\hbar \omega_{\mathrm{L}}},
\end{aligned}
$$

with intermediate states $|n\rangle$. To relate our result in SI units to atomic units, where $\hbar=a_{0}=e=m_{\mathrm{e}}=1$, one has to apply the conversion

$$
\beta_{g e}(\text { a.u. })=\beta_{g e}(\mathrm{SI}) \times \frac{\hbar^{2}}{m_{\mathrm{e}} a_{0}^{4} \alpha},
$$

in which these constants are reinstated, resulting in a value of $\beta_{g e}=7.85366$ atomic units. In Ref. [57], the authors define

$$
D\left[J_{0}\right]=\frac{3}{2} \sum\left(1+P_{12}\right) \frac{\hat{\mathbf{e}}_{1} \cdot\left\langle 2 S\left|\mathbf{r} / a_{0}\right| n\right\rangle\left\langle n\left|\mathbf{r} / a_{0}\right| 1 S\right\rangle \cdot \hat{\mathbf{e}}_{2}}{\nu(n)-\nu(1 s)-\nu_{2}}
$$

as the two-photon transition probability amplitude and obtain a value of -11.7805 atomic units, which differs precisely by a factor of $-\frac{3}{2}$ from our result. The opposite sign is obviously just a consequence of a different definition of the Green's function. The operator $P_{12}$ interchanges the polarization vectors $\hat{\mathbf{e}}_{1}$ and $\hat{\mathbf{e}}_{2}$ and the frequencies of the two photons. In that work, the light fields are treated more generally to have different frequencies. For distinguishable photons, there are two different ways to excite the atom, one path with first lowfrequency and then high-frequency photon absorption and another with first high-frequency and then low-frequency photon absorption.

To reduce definition (E3) to the case of equal polarizations and equal frequencies, as considered in this paper, we
TABLE XIII. Comparison of squared transition matrix elements $M$, as defined in Ref. [59].

\begin{tabular}{lcc}
\hline \hline Literature source & Transition & $M$ \\
\hline Tung et al. $[59]$ & $1 S-3 S$ & 4.2812 \\
This work & $1 S-3 S$ & 4.5822 \\
Tung et al. [59] & $2 S-6 S$ & 261.44 \\
This work & $2 S-6 S$ & 216.4420 \\
\hline \hline
\end{tabular}

have to set the polarization vectors $\hat{\mathbf{e}}_{1}=\hat{\mathbf{e}}_{2}=\hat{\mathbf{e}}_{z}$ and we have to omit the part where the polarizations and frequencies are interchanged, because the photons are indistinguishable. Then the manifest prefactor of $\frac{3}{2}$ in Eq. (E3) explains the numerical difference as being only a matter of definition, while the physical results agree. This value of $D\left[J_{0}\right]$ is cited and used by Ref. [58]; therefore the same considerations apply there.

\section{1S-3S transition}

The result of this work for the $1 S-3 S$ transition matrix element, reads $\beta_{g e}=1.00333 \times 10^{-5} \mathrm{~Hz} /\left(\mathrm{W} / \mathrm{m}^{2}\right.$ ) (see Table II), or $\beta_{g e}=2.14061$ when converted to atomic units. This equals the value given in Ref. [3]. Also the other $S-S$ transition matrix elements calculated there, namely, for the $2 S-6 S$ and $2 S-8 S$ transitions, are equal to the results presented here, if converted according to Eq. (E2).

In Ref. [59], Table 20, the squares of the transition matrix elements, called $M$, for the transitions $1 S-n S, 1 S-n D, 2 S-n S$, and $2 S-n D$ with $n$ up to 6 are given. Out of these 18 squared matrix elements (given in atomic units), our results are in agreement with 16 values. The cases in which differences occur are the values for the $1 S-3 S$ and the $2 S-6 S$ transitions (see Table XIII). The value for $M(1 S-3 S)$ given by Tung et $a l$. is equal to twice the transition matrix element from this work (2.140 61), not the square, so it is likely that Tung et al. inadvertently doubled their correct result for the transition matrix element instead of squaring it. The second inconsistency might be explained by a twist of digits.

In an even older study [60], the authors work in the velocity gauge, as opposed to the length gauge used in all articles considered previously. Tung et al. [59] agree with their results for the absorption cross section, which is related to the transition matrix element. This supports the assumption that Tung et al. have obtained matrix elements in agreement with the results presented here, while the numerical differences originated in the compilation process.

\section{Light shifts}

The complete set of results for the light shifts of $S$ states, for transitions where the $2 S$ state acts as the ground state (see 
Table V), agree with Table Ic of Ref. [6]. In that work, where atomic units are used, only the real parts of the dynamic Stark shifts are presented; the light shift of $20 S$ in the $2 S-20 S$ transition, for example, is 261.18 atomic units in Ref. [6], which can be converted via relation (E2) into $\beta_{\mathrm{ac}}(20 S)=1.22420 \times 10^{-3} \mathrm{~Hz} /\left(\mathrm{W} / \mathrm{m}^{2}\right)$ in agreement with the value given in Table $\mathrm{V}$. The light shifts of the $D$ states given in the same table in Ref. [6] agree with the values obtained in this paper, when the atomic FS and HFS are unresolved.
[1] F. Biraben et al., in The Hydrogen Atom: Precision Physics of Simple Atomic Systems, edited by S. G. Karshenboim, F. S. Pavone, G. F. Bassani, M. Inguscio, and T. W. Hänsch (Springer, Berlin, 2001), pp. 18-41.

[2] M. Fischer et al., Phys. Rev. Lett. 92, 230802 (2004).

[3] B. de Beauvoir, C. Schwob, O. Acef, L. Jozefowski, L. Hilico, F. Nez, L. Julien, A. Clairon, and F. Biraben, Eur. Phys. J. D 12, 61 (2000).

[4] M. Stobbe, Ann. Phys. 7, 661 (1930).

[5] J. C. Garreau, M. Allegrini, L. Julien, and F. Biraben, J. Phys. (France) 51, 2263 (1990).

[6] J. C. Garreau, M. Allegrini, L. Julien, and F. Biraben, J. Phys. (France) 51, 2275 (1990).

[7] J. C. Garreau, M. Allegrini, L. Julien, and F. Biraben, J. Phys. (France) 51, 2293 (1990).

[8] V. Yakhontov, R. Santra, and K. Jungmann, J. Phys. B 32, 1615 (1999).

[9] V. Yakhontov and K. Jungmann, Z. Phys. D: At., Mol. Clusters 38, 141 (1996).

[10] B. de Beauvoir, C. Schwob, O. Acef, L. Jozefowski, L. Hilico, F. Nez, L. Julien, A. Clairon, and F. Biraben, Eur. Phys. J. D 12, 61 (2000).

[11] S. Chu, A. P. Mills, A. G. Yodh, K. Nagamine, Y. Miyake, and T. Kuga, Phys. Rev. Lett. 60, 101 (1988).

[12] K. Danzmann, M. S. Fee, and S. Chu, Phys. Rev. A 39, R6072 (1989).

[13] K. Jungmann et al., Z. Phys. D: At., Mol. Clusters 21, 241 (1991).

[14] V. Meyer et al., Phys. Rev. Lett. 84, 1136 (2000).

[15] Ch. Gohle, Th. Udem, M. Herrmann, J. Rauschenberger, R. Holzwarth, H. A. Schuessler, F. Krausz, and T. W. Hänsch, Nature (London) 436, 234 (2005).

[16] M. Niering et al., Phys. Rev. Lett. 84, 5496 (2000).

[17] C. Schwob, L. Jozefowski, B. de Beauvoir, L. Hilico, F. Nez, L. Julien, F. Biraben, O. Acef, J. J. Zondy, and A. Clairon, Phys. Rev. Lett. 82, 4960 (1999).

[18] N. Kolachevsky, M. Haas, U. D. Jentschura, M. Herrmann, M. Fischer, R. Holzwarth, Th. Udem, C. H. Keitel, and T. W. Hänsch (unpublished).

[19] B. J. Shortt, P. J. M. van der Burgt, and F. Giammanco, Laser Phys. 12, 1402 (2002).

[20] P. Zoller, Phys. Rev. A 19, 1151 (1979).

[21] W. Demtröder, Laser Spectroscopy, 3rd ed. (Springer, Berlin, 2003).

[22] G. S. Agarwal, Phys. Rev. Lett. 37, 1383 (1976).

[23] M. Scully and M. Zubairy, Quantum Optics (Cambridge University Press, Cambridge, U.K., 1999).

[24] W. Vogel, D.-G. Welsch, and S. Wallentowitz, Quantum Optics: An Introduction (Wiley-VCH, Berlin, 2001).

[25] F. Bloch and A. Siegert, Phys. Rev. 57, 522 (1940).
[26] H. F. Beyer, H.-J. Kluge, and V. P. Shevelko, X-Ray Radiation of Highly Charged Ions (Springer, Berlin, 1997).

[27] B. L. Beers and L. Armstrong, Phys. Rev. A 12, 2447 (1975).

[28] For the lines inverted in the center (see, e.g., Fig. 4 for long interaction times) we use the distance of the outermost half values as the linewidth.

[29] U. D. Jentschura, Phys. Rev. A 69, 052118 (2004).

[30] J. D. Cresser, A. Z. Tang, G. J. Salamo, and F. T. Chan, Phys. Rev. A 33, 1677 (1986).

[31] V. Florescu, I. Schneider, and I. N. Mihailescu, Phys. Rev. A 38, 2189 (1988).

[32] H. A. Bethe and E. E. Salpeter, Quantum Mechanics of Oneand Two-Electron Atoms (Springer, Berlin, 1957).

[33] W. H. Press, B. P. Flannery, S. A. Teukolsky, and W. T. Vetterling, Numerical Recipes in C: The Art of Scientific Computing, 2nd ed. (Cambridge University Press, Cambridge, U.K., 1993).

[34] P. M. Koch, Phys. Rev. Lett. 41, 99 (1978).

[35] D. H. Kobe, J. Phys. B 16, 1159 (1983).

[36] M. Gavrila and A. Costescu, Phys. Rev. A 2, 1752 (1970).

[37] K. Pachucki, Ann. Phys. (N.Y.) 226, 1 (1993).

[38] S. Salomonson and P. Öster, Phys. Rev. A 40, 5559 (1989).

[39] V. Yakhontov, Phys. Rev. Lett. 91, 093001 (2003).

[40] R. A. Swainson and G. W. F. Drake, J. Phys. A 24, 79 (1991).

[41] R. A. Swainson and G. W. F. Drake, J. Phys. A 24, 95 (1991).

[42] R. A. Swainson and G. W. F. Drake, J. Phys. A 24, 1801 (1991).

[43] A. R. Edmonds, Angular Momentum in Quantum Mechanics (Princeton University Press, Princeton, NJ, 1957).

[44] C. Cohen-Tannoudji, J. Dupont-Roc, and G. Grynberg, AtomPhoton Interactions (Wiley, New York, 1992).

[45] M. Haas, U. D. Jentschura, and C. H. Keitel, Am. J. Phys. 74, 77 (2006).

[46] H. Bateman, Higher Transcendental Functions (McGraw-Hill, New York, 1953), Vol. 1.

[47] S. Wolfram, Mathematica-A System for Doing Mathematics by Computer (Addison-Wesley, Reading, MA, 1988).

[48] J. C. de Vries, Ph.D. thesis, MIT Report No. 658, 2002 (unpublished).

[49] J. Evers, U. D. Jentschura, and C. H. Keitel, Phys. Rev. A 70, 062111 (2004).

[50] M. Herrmann, Th. Udem, C. Gohle, N. Kolachevsky, T. W. Hänsch, D. Leibfried, D. Wineland, M. Haas, U. D. Jentschura, and C. H. Keitel (unpublished).

[51] C. E. Theodosiou, L. Armstrong, M. Crance, and S. Feneuille, Phys. Rev. A 19, 766 (1979).

[52] J. L. F. de Meijere and J. H. Eberly, Phys. Rev. A 17, 1416 (1978).

[53] G. S. Agarwal, S. L. Haan, K. Burnett, and J. Cooper, Phys. Rev. Lett. 48, 1164 (1982). 
[54] G. S. Agarwal, S. L. Haan, and J. Cooper, Phys. Rev. A 29, 2552 (1984).

[55] S. A. Lee, Ph.D. thesis, Stanford University, M. L. Report No. 2460, 1975 (unpublished).

[56] U. D. Jentschura and C. H. Keitel, Ann. Phys. (N.Y.) 310, 1 (2004).

[57] F. Bassani, J. J. Forney, and A. Quattropani, Phys. Rev. Lett.
39, 1070 (1977).

[58] M. G. Boshier, P. E. G. Baird, C. J. Foot, E. A. Hinds, M. D. Plimmer, D. N. Stacey, J. B. Swan, D. A. Tate, D. M. Warrington, and G. K. Woodgate, Phys. Rev. A 40, 6169 (1989).

[59] J. H. Tung, A. Z. Tang, G. J. Salomo, and F. T. Chan, J. Opt. Soc. Am. B 3, 837 (1986).

[60] Y. Gontier and M. Trahin, Phys. Lett. 36A, 463 (1971). 